\title{
Impacto de los estándares internacionales de información financiera en la calidad contable: evidencia desde Latinoamérica y el Caribe ${ }^{1}$
}

\section{The impact of international financial reporting standards on accounting quality: Evidence from Latin America and the Caribbean}

\author{
Juan Camilo Cardona Montoya ${ }^{1 *}$, Andrés Felipe Gómez Sánchez², \\ Abel María Cano Morales ${ }^{3}$ \\ ${ }^{1}$ Universidad de San Buenaventura, Colombia \\ ${ }^{2}$ Universidad de San Buenaventura, Colombia \\ ${ }^{3}$ Universidad de Medellín, Colombia
}

Fecha de recepción: 7.Septiembre.2017; Fecha de aceptación: 28.Junio.2018

Disponible en internet el: 13 de septiembre de 2019

\section{Resumen}

Este artículo examina el impacto de la aplicación de los Estándares Internacionales de Información Financiera (IFRS) en la calidad contable (calidad del reporte financiero) de las economías latinoamericanas y del Caribe, respecto a su marco normativo local (Local GAAP); usando una muestra de compañías cotizadas en Latinoamérica y el Caribe durante los periodos 2006-2016, se valora la calidad contable bajo tres criterios de medición: la manipulación del resultado, el reconocimiento oportuno de pérdidas (atributos contables) y la relevancia valorativa (atributos de mercado). Los hallazgos sugieren un mejoramiento parcial de la calidad contable, evidenciado a través de una disminución en el nivel de manipulación del resultado sólo después de varios años de aplicación de IFRS. Sin embargo, no se

\footnotetext{
*Autor para correspondencia

Correo electrónico: juan.cardona@usbmed.edu.co (J.C. Cardona Montoya)

La revisión por pares es responsabilidad de la Universidad Nacional Autónoma de México.

http://dx.doi.org/10.22201/fca.24488410e.2018.1669

0186- 1042/C 2019 Universidad Nacional Autónoma de México, Facultad de Contaduría y Administración. Este es un artículo

Open Access bajo la licencia CC BY-NC-SA (https://creativecommons.org/licenses/by-nc-sa/4.0/)

${ }^{1}$ Scientific and technological research paper derived from the research project "Impact of International Financial Reporting Standard on accounting quality for Latin American capital markets II", financed by the University of San Buenaventura Medellín, Colombia, 2018.
} 
evidencia mayor oportunidad al reconocer grandes pérdidas, al tiempo que, la información contable generada bajo los estándares IFRS, no es valorada con mayor utilidad por los agentes de mercado.

Código JEL: M41, M42, M48

Palabras Clave: Adopción IFRS; Alisamiento del resultado; Calidad contable; Manipulación del resultado;

Relevancia valorativa

\begin{abstract}
This paper examines the impact of the application of International Financial Reporting Standards (IFRS) on accounting quality (financial report quality) of Latin American and Caribbean economies, regarding to their local accounting regulatory standards (Local GAAP). By using a sample of listed companies in Latin America and the Caribbean from 2006 to 2016, accounting quality is assessed under three measurement criteria: earnings management, timely loss recognition (accounting attributes) and value relevance (market attributes). The findings suggest a partial accounting quality improvement; it is showed through a decrease on the level of earnings management only after several years of the IFRS application. However, there is no evidence of a greater opportunity to recognize large losses; furthermore, the generated accounting information under IFRS is not valued, with greater usefulness by market agents.
\end{abstract}

JEL Code: M41, M42, M48

Keywords: IFRS adoption; Earnings smoothing; Accounting quality; Earnings management; Value relevance

\title{
Introducción
}

Cuantos más países aplican los Estándares Internacionales de Información Financiera (en adelante IFRS, por sus siglas en inglés), se hace mayor la necesidad y el interés del mundo académico contable y de los organismos reguladores a nivel nacional e internacional, por conocer en profundidad el resultado de su aplicación en función de las características institucionales de cada país adoptante. Son numerosos los trabajos que ponen de manifiesto la importancia de comprobar si la adopción de una normativa contable internacional común, como es el caso de las IFRS, es igualmente aplicable en una gran diversidad de naciones que se caracterizan por sus diferencias políticas, económicas e institucionales; sin olvidar, que las IFRS están basadas en las prácticas contables de países anglosajones, en su mayoría de las prácticas de países como Reino Unido y los Estados Unidos de América (Hove, 1990). Investigaciones previas analizan si estas normas son apropiadas para otros países distintos a los de origen anglosajón, sin embargo, la relevancia de las IFRS, es todavía un asunto de interés por muchos académicos y reguladores para los países en vía de desarrollo. 
Por lo anterior, esta investigación tiene por objetivo analizar si la aplicación de las IFRS, contribuye al mejoramiento de la calidad de los estados financieros (expresión de medición de la calidad contable) para los países latinoamericanos y del Caribe. Concretamente, se valora si el uso de estos estándares se asocia con un mejoramiento en la calidad de la información contable respecto a la aplicación de los principios contables generalmente aceptados en cada país (en adelante Local GAAP, por sus siglas en inglés); esto se evidencia a través de una menor manipulación del resultado, mayor oportunidad en el reconocimiento de pérdidas y mayor relevancia valorativa de las magnitudes contables en los mercados de capitales durante el periodo de aplicación de IFRS, respecto al periodo de aplicación de las normas locales.

El resto del artículo se encuentra organizado de la siguiente manera. En la segunda sección se revisa la literatura previa en torno al desarrollo y aplicación de las expresiones de medición de la calidad contable y la influencia de los factores institucionales como determinantes de dicha calidad, además de presentarse el planteamiento de las hipótesis para este estudio; la tercera y cuarta sección describe el diseño metodológico y la selección y distribución de la muestra, respectivamente; la quinta sección presenta los resultados empíricos del estudio, mientras que la sexta, los resultados obtenidos bajo un análisis de sensibilidad. Finalmente, la última sección presenta las conclusiones de esta investigación.

\section{Revisión de la literatura y definición de hipótesis}

\section{Expresiones de medición de la calidad contable y su aplicabilidad a nivel internacional}

Las primeras manifestaciones teóricas sobre el concepto multifacético de la calidad contable basado en la noción de la manipulación del resultado (earnings management), tuvieron su origen durante los años 80 y 90, por ejemplo, Schipper (1989), define la manipulación del resultado como la intervención discrecional por parte de la gerencia sobre el proceso de reporte de los estados financieros; años más tarde, esta concepción fue complementada por Healy y Wahlen (1999), quienes sugieren que dicha intervención conduce a error a los grupos de interés (stakeholders) sobre el desempeño económico de la empresa. El concepto de la manipulación del resultado fue referido a su vez, a través de un indicador a fin a la manera o forma de intervención discrecional ejercida por la gerencia sobre las cuentas de resultado, dicho indicador es descrito como el alisamiento del resultado (earnings smoothing), que de acuerdo con Trueman y Titman (1988), se presenta cuando la dirección de las compañías toma acciones para reducir las fluctuaciones en el beneficio neto reportado.

Posteriormente, dichas manifestaciones teóricas de la calidad contable, visto desde el efecto combinado de los elementos del sistema de reporte sobre las variables contables principales, fueron operacionalizadas y desarrolladas como expresiones de medición (proxies) 
durante los años 2003 a 2008, y junto a éstas, fueron utilizadas las expresiones relacionadas con el nivel de conservadurismo contable y del papel informativo de la contabilidad (relevancia valorativa). Leuz et al. (2003), documentaron una primera aproximación al elaborar y aplicar a nivel internacional, nuevas expresiones para la medición de la calidad contable, basadas en la relación de la variabilidad de las ganancias con la variabilidad de los ajustes por devengo, y la correlación entre estos últimos y los flujos de efectivo operativos, además de otras mediciones; mediciones que posteriormente fueron empleadas y complementadas por Lang et al. (2003) y Lang et al. (2006), para comparar los efectos de la calidad contable entre las empresas que cotizan en las bolsas de los estados unidos respecto a las que no, y entre las empresas cotizadas locales y cotizadas extranjeras en los mercados estadounidenses con reconciliación de ganancias, respectivamente.

Estas expresiones posteriormente, se han convertido en uno de los instrumentos con mayor acogida para comparar si la calidad del reporte financiero varía con el uso de los estándares IFRS, además de otros marcos normativos contables como los estándares generalmente aceptados por los Estados Unidos de América (US GAAP, por sus siglas en inglés). Los primeros trabajos reportados bajo esta línea, fueron los conducidos por Barth et al. (2006) y Barth et al. (2008), quienes han documentado un menor nivel de manipulación del resultado para aquellas empresas que aplican US GAAP respecto a aquellas que aplican IAS (ahora IFRS), y una menor manipulación de las ganancias, mayor oportunidad en el reconcomiendo de pérdidas y mayor relevancia valorativa para aquellas compañías que voluntariamente aplican IFRS a nivel internacional, respectivamente.

A partir de estas propuestas, la literatura contable basada en la calidad de los estados financieros y especialmente la relacionada con los efectos de la aplicación de las IFRS, se ha desarrollado vertiginosamente con tres alternativas de medición, dos de ellas basadas en atributos contables (la manipulación del resultado y el reconocimiento oportuno de pérdidas) y la restante basada en atributos de mercado (la relevancia valorativa).

Aplicando estas alternativas, Paananen (2008) y Paananen y Lin (2009), concluyen que la calidad del reporte financiero no mejora significativamente en Suecia y Alemania respectivamente, por el contrario, presenta una disminución durante los primeros años de aplicación de la normativa IFRS; por su parte, Christensen et al. (2015), Günther et al. (2009) y Lin et al. (2012), reportan resultados similares para el caso de las empresas alemanas obligadas a aplicar dichos estándares, sugiriendo un incremento en el nivel de manipulación del resultado y una disminución en la relevancia valorativa; sin embargo, para las empresas alemanas que voluntariamente aplican IFRS, los estudios conducidos por Christensen et al. (2015) y Günther et al. (2009) indican una disminución en el nivel de alisamiento del resultado y un incremento al incorporar oportunamente las pérdidas; y únicamente Christensen et al. (2015), revelan 
un mejoramiento en la relevancia de valor de las magnitudes contables en los mercados de capitales para estas empresas.

Los estudios de la calidad contable fueron aplicados simultáneamente en otros países europeos, siendo los casos de Reino Unido (Iatridis 2008, 2010), Grecia (Dimitropoulos et al., 2013) y Rumania (Brad et al., 2014), en donde se evidenció una disminución en el nivel de alisamiento del resultado durante los periodos de aplicación obligatoria de IFRS, además de un incremento en la oportunidad al reconocer grandes pérdidas y un mejoramiento en el papel informativo de la información contable para los dos primeros casos en mención; estos resultados son también extensibles a la aplicación voluntaria de las IFRS en las empresas de Reino Unido (Iatridis 2008, 2010), y para el caso Griego según el estudio conducido por Iatridis y Rouvolis (2010), evidencian un mejoramiento en los aspectos de manipulación del resultado y relevancia valorativa para estas últimas empresas a partir del segundo periodo de aplicación de IFRS y en adelante, con relación al primer periodo de aplicación de estos estándares.

Respecto a los estudios multi-país de la calidad contable puede decirse que se obtienen resultados diversos, siendo el caso de Zeghal et al. (2012), quienes documentan una disminución (aumento) en los atributos de mercado (atributos contables) para 15 países de la unión europea; por el contrario, el estudio conducido por Devalle et al. (2010) no evidenció un mejoramiento en estos últimos atributos puesto que el alisamiento del resultado (la oportunidad en el reconocimiento de perdidas) no disminuyó (no incrementó) significativamente para las 5 economías más grandes de Europa (España, Francia, Alemania, Italia y Reino Unido). En esta misma dirección, el estudio conducido por Aubert y Grudnitski (2011) aplicado sobre 13 países miembros de la unión europea, tampoco evidenció algún incremento significativo en la relevancia valorativa durante el periodo de post-adopción, sin embargo, Piot et al. (2010) lograron confirmar, para 22 países europeos, una disminución en el nivel de conservadurismo contable.

En este orden de ideas, Ahmed et al. (2013), al evaluar la calidad del reporte financiero desde 29 países que obligatoriamente adoptaron las IFRS durante el año 2005, indican una disminución en dicha calidad, evidenciado a partir de un incremento (disminución) en la manipulación del resultado (oportunidad en el reconocimiento de grandes pérdidas) respecto a aquellos países no adoptantes.

Capkun et al. (2016) Además, hallan evidencia sobre un incremento en la manipulación del resultado para aquellos países obligados a adoptar las IFRS desde el año 2005, al igual que para aquellos que las adoptaron voluntariamente antes de dicho año e hicieron el cambio a la versión IFRS 2005, sugiriendo que la versión de las IFRS emitida por el IASB, durante el año indicado, permitió la elección de alternativas contables aptas por los directivos para manipular el resultado contable. 
Los estudios de la calidad contable bajo la metodología empleada por Barth et al. (2008), fueron extendidos posteriormente, a los países americanos y orientales, autores como Cardona Montoya y Cano Morales (2017), al evaluar el efecto de la entrada en vigencia de las IFRS en Canadá, indican un incremento en el nivel de conservadurismo contable y en la capacidad informativa de las magnitudes contables para su mercado de capitales; Zhou et al. (2009) por su parte, hallan una disminución en el nivel de manipulación del resultado para las compañías chinas que aplican IFRS respecto a las empresas que no lo hacen, aunque no evidencian mayor oportunidad al reconocer pérdidas; Liu et al. (2011), aparte de documentar menor manipulación del resultado, reportan un incremento en la relevancia valorativa de la información contable para las compañías chinas obligadas a implementar IFRS en el año 2007, analizado frente a su periodo de pre-adopción.

Hallazgos similares a los anteriores, fueron obtenidos por Temiz y Güleç (2017), Abdullah et al. (2017) y Fuad y Wijanarto (2017) en sus estudios para las empresas cotizadas no financieras de Turquía, Malasia e Indonesia, respectivamente, en donde reportan un incremento en la relevancia valorativa para el primer caso y una disminución en el nivel de manipulación del resultado para las restantes economías durante los periodos de aplicación de IFRS respecto a sus correspondientes periodos precedentes. Finalmente, Chua et al. (2012), concluyen sobre un incremento en la calidad del informe financiero al hallar una disminución en el alisamiento del resultado, un incremento en la frecuencia oportuna al reconocer las pérdidas y un incremento en la capacidad informacional de la contabilidad para aquellas compañías australianas obligadas a aplicar IFRS desde el año 2005.

Respecto a literatura de la calidad contable bajo el concepto de la relevancia valorativa (value relevance), fue desarrollada a través de métodos alternativos para constatar la capacidad informativa de las magnitudes contables en los mercados de capitales (Hellstrom, 2006), siendo los modelos propuestos por Bartov et al. (2005), Basu (1997) y Ohlson (1995), las alternativas presentes en la mayoría de estudios aplicados y relacionados hasta ahora; el modelo propuesto por Ohlson (1995) por ejemplo, propone que las variaciones en el precio de la acción podrían ser explicadas por los importes del resultado contable y del valor en libros del patrimonio reportados al mercado; Basu (1997), dirige su análisis hacia el concepto del conservadurismo del resultado, bajo la concepción que las rentabilidades negativas (malas noticias) son incorporadas con mayor oportunidad en el resultado contable, respecto a la oportunidad con que se incorporan las rentabilidades positivas (buenas noticias); por su parte, Bartov et al. (2005), proponen su medición relacionando la rentabilidad bursátil con el resultado contable del periodo, y al aplicarla sobre un grupo de empresas alemanas, indican una mayor capacidad informacional de las ganancias obtenidas a partir de las US GAAP respecto a las obtenidas con la aplicación de las IFRS. 


\section{Estudios sobre la calidad contable y los factores institucionales en Latinoamérica}

\section{Estudios sobre la calidad contable en Latinoamérica}

Durante las últimas décadas, el uso obligatorio de las IFRS por la mayoría de países latinoamericanos, permitió la aplicación de varios estudios tendientes en analizar el impacto de esta normativa sobre la calidad contable, principalmente de las empresas cotizadas en Brasil, México y Chile, aunque los hallazgos documentados hasta ahora han resultado ser un tanto contradictorios. Vieira et al. (2011) por ejemplo, al utilizar la metodología empleada por Barth et al. (2008), evidencian una disminución en el nivel de manipulación del resultado y un mejoramiento en la relevancia valorativa de la contabilidad para aquellas empresas brasileñas que adoptaron parcialmente las IFRS durante el año 2008; Klann y Beuren (2015) por el contrario, precisan un incremento en la manipulación del resultado en los periodos de adopción plena de las IFRS para estas mismas compañías durante el año 2010, mientras que Pelucio-Grecco et al. (2014), analizando la calidad contable durante los años 2006-2011, concluyen que la transición a las IFRS restringe el nivel de manipulación del resultado después de completar la implementación respecto a los periodos previos a dichas normas.

Los resultados obtenidos en el estudio anterior han sido reforzados recientemente por da Silva et al. (2017) para el sector eléctrico, quienes al analizar los estados financieros de las empresas de este sector entre los años 2003 a 2014, reportan una reducción en el nivel de alisamiento del resultado después de la adopción de las IFRS, concluyendo sobre un mejoramiento en la calidad contable visto desde una disminución en el nivel de manipulación del resultado.

Para el caso mexicano, Conesa et al. (2011), al analizar la adaptación de los GAAP de este país respecto a las IFRS sobre los años 1997-2009, sugieren una disminución en el nivel de manipulación contable, sin embargo, Palacios Manzano y Martínez Conesa (2014), no sugieren una disminución en dicho nivel de manipulación al encontrar que, para el periodo 1997-2008, la magnitud de los ajustes por devengo discrecionales (expresión de medición de la calidad contable), no disminuye con el uso de las IFRS en las compañías mexicanas no financieras cotizadas en el NYSE.

Por su parte Santana et al. (2014), indican que las empresas latinoamericanas reportan un menor nivel de calidad contable durante los años 2011 y 2012, al comparar la magnitud de los ajustes por devengo discrecionales entre las empresas cotizadas en Brasil y Chile respecto a Reino Unido y Australia, y respecto a Francia y Alemania, aunque Cardona Montoya (2018) y Melgarejo (2017), analizando el impacto de la aplicación de las IFRS durante los años 2006 a 2014 y 1997 a 2015 respectivamente, sugieren un incremento en la calidad contable para 25 países latinos y del Caribe y para las 5 principales economías latinas respectivamente, evidenciando que el uso de las IFRS impacta negativamente la magnitud de los ajustes por 
devengo discrecionales, además que, el último trabajo en mención, identifica una mayor nivel de asociación entre las ganancias contables reportadas y la cotización bursátil durante el periodo de aplicación de estos estándares.

\section{Factores institucionales y su eficacia en Latinoamérica}

Recientes estudios en la literatura contable dirigen su atención en identificar aquellos factores institucionales determinantes de la calidad del reporte de información financiera y de revelación (Ball, 2001; Ball et al., 2003; Ball \& Shivakumar, 2005; Burgstahler et al., 2006; Hail et al., 2010; Leuz et al., 2003), en los que además, aparte de considerar los estándares IFRS como un factor institucional, reconocen no son el único elemento por sí solo, que condiciona el comportamiento de las prácticas de reporte financiero entre países (Leuz \& Wysocki, 2016), especialmente el comportamiento en el nivel de manipulación del resultado (Houqe et al., 2012; Leuz et al., 2003; Reverte, 2008).

Varios estudios advierten sobre la presencia de posibles interdependencias entre los mismos factores institucionales, además de ser todos ellos complementarios para afectar la calidad del reporte financiero (Leuz, 2010; Leuz \& Wysocki, 2016; Wysocki, 2011); adicionalmente, la literatura hace un llamado por dar respuesta al alto interés de académicos y reguladores del mercado por identificar aquellas instituciones que son necesarias, suficientes o más importantes para el desarrollo de los mercados de capitales entre países (Wysocki, 2011). Estudios previos, como los conducidos por Kothari (2000) y Ball (2001), ya develaban otras instituciones distintas del sistema contable, que podrían condicionar el desarrollo de los mercados económicos y de capitales en un país, por ejemplo, instituciones de tipo legal, económico y regulatorio; sin embargo, estudios más recientes, identifican y procesan sobre 35 países, una amplia variedad de factores institucionales, además de los anteriores, otros de tipo sociológico y geopolítico, y que de alguna manera afectan la calidad de la información contable (Isidro et al., 2016).

Respecto a la presencia de estas instituciones en Latinoamérica y el Caribe, tal como lo revelan los estudios multi-país, presentan marcadas diferencias respecto a las instituciones presentes en el resto de países (Leuz, 2010), y por tanto, en sintonía con la literatura previa, afectaría la calidad de la información contable durante la aplicación obligatoria de las IFRS. Precisamente, al hacer seguimiento al grado de eficacia de algunas de las instituciones presentes para la región latina, se identifican las siguientes instituciones: los niveles de independencia judicial, de independencia del consejo de administración y de protección a los derechos de los accionistas minoritarios, los niveles de financiación a través de los mercados de capitales y de desarrollo de los mismos, y el grado de efectividad de los mecanismos de refuerzo y control (enforcement) sobre los estándares de información financiera y de auditoría. 
El nivel de independencia judicial, como factor de seguridad y respaldo a los derechos de propiedad de los inversores (La Porta et al., 2004), resulta ser en media, significativamente menor para los países de tradición de derecho civil-francés (en la que se encuentra la totalidad de los países latinoamericanos) respecto a los demás grupos de países de derecho civil (alemán y escandinavo) y de derecho común (anglosajón) (Porta et al., 1998), lo cual impacta negativamente la transparencia financiera de las empresas (Bushman et al., 2004), además de presentar prácticas contables menos conservaduristas respecto a las prácticas de países con mayor independencia judicial (Bushman \& Piotroski, 2006); estudios más recientes, como el dirigido por Leuz (2010), ubica a la región latinoamericana en las posiciones más bajas del índice de estado de derecho después de la regiones del lejano oriente y del mediterráneo en su orden, y únicamente por encima de la región africana.

Respecto a la independencia del consejo de administración y al nivel de protección de los derechos de los accionistas minoritarios, varios estudios han sugerido que los niveles de manipulación del resultado son susceptibles de aumento, en países en donde sus empresas cuentan con la presencia de un menor número de consejeros independientes (Ebrahim, 2007; Klein, 2002; Peasnell et al., 2005) y presentan un mayor grado de concentración de la propiedad accionarial (Reverte, 2008) respectivamente; si bien La Porta et al. (2000), describen que aquellos países con tradición de derecho civil-francés reportan un menor nivel de protección a los derechos de los inversores y por ende, menor calidad en las medidas de gobierno corporativo, recientemente el Índice de Medición de Competitividad Global, indica un leve mejoramiento en media, respecto a la eficacia de los consejos de administración y en los niveles de protección de los intereses minoritarios en la mayoría de países latinoamericanos (WEF, 2017); adicionalmente, el índice de protección a los accionistas minoritarios calculado en el estudio conducido por Leuz (2010), revela una posición de la región latinoamericana por encima de las regiones del Mediterráneo y de la Europa Oriental.

Por otra parte, los niveles de financiación a través de los mercados de capitales (como expresión de medición del tamaño del mercado) y de regulación de dichos mercados, son considerados para tener una relación inversa con los niveles de manipulación del resultado según los estudios de Halabi y Yi (2015) y Reverte (2008), respectivamente. Si bien los mercados de capitales latinoamericanos han sido clasificados como mercados emergentes (Leuz, 2010), reportan en media, una mejoría en ambas instituciones según el Índice de Medición Global para la mayoría de sus países (WEF, 2017), mejoría que es documentada por Leuz (2010) en su índice de regulación de valores, estando incluso por encima de la región mediterránea, africana y de la Europa Oriental; adicionalmente, en el estudio multi-país conducido por Houqe et al. (2012), en el que incluyen los 7 principales mercados de capitales latinoamericanos, constataron la relación inversa antes indicada, entre los niveles de regulación de dichos mercados y de manipulación del resultado (medido a través de la magnitud de los ajustes por 
devengo discrecionales) durante los periodos de aplicación de IFRS.

Finalmente, referente al grado de efectividad de los mecanismos de refuerzo y control sobre los estándares de información financiera y sobre los estándares de auditoría, Halabi y Yi (2015) demuestran una relación inversa entre la primera de estas instituciones y la magnitud de los ajustes por devengo discrecionales durante la aplicación de los estándares IFRS a nivel multi-país, mientras que Francis et al. (2003), sugieren una relación directa entre la segunda institución y la calidad del reporte financiero. Y aunque algunos estudios han reportado reacciones negativas en los mercados de capitales con la adopción de las IFRS en países donde la primera institución es calificada como débil (Armstrong et al., 2010), la región latinoamericana se caracteriza por mejorar en media, sus posiciones para ambas instituciones en la mayoría de sus países durante los últimos años, según el Índice de Competitividad Global (WEF, 2017), mejora que es evidencia por Houqe et al. (2012) en su estudio multi-país (en el que incluyen varios países latinoamericanos), al constatar un menor nivel de manipulación cuando ambas instituciones son calificadas como rigurosas.

\section{Definición de hipótesis}

Con base en los hallazgos documentados en la literatura internacional y en especial en los relacionados hasta ahora para la región latinoamericana, se plantean las siguientes hipótesis: H1: Se espera que durante los periodos de aplicación de IFRS, disminuya significativamente el nivel de manipulación del resultado respecto a los periodos de aplicación de GAAP locales en la región Latinoamericana y del Caribe.

H2: Se espera que durante los periodos de aplicación de IFRS, aumente significativamente la oportunidad al reconocer las pérdidas respecto a los periodos de aplicación de GAAP locales en la región latinoamericana y del Caribe.

H3: Se espera que durante los periodos de aplicación de IFRS, la información contable presente un incremento significativo en la capacidad informativa en los mercados de capitales latinoamericanos y del Caribe respecto a los periodos de aplicación de GAAP locales.

\section{Metodología}

En este estudio se operacionaliza el concepto de la calidad contable a través de la calidad de los reportes financieros y este a su vez, a través de tres expresiones (proxies) relacionadas con la calidad del resultado reportado, las cuales son globalmente empleadas en la literatura contable, dos de ellas basadas en atributos contables (la manipulación del resultado y el reconocimiento oportuno de pérdidas) y la restante, basada en datos del mercado (la relevancia 
valorativa), todas ellas fortalecen los hallazgos encontrados respecto al concepto multifacético de la calidad contable.

Por tanto, las expresiones utilizadas para examinar la calidad contable no solamente reflejan los efectos atribuibles al marco normativo contable aplicado, también a los demás elementos del sistema contable, incluyendo además la interpretación que los directivos realicen sobre las normas, los mecanismos de refuerzo (enforcement) utilizados por cada país para garantizar la correcta aplicación del marco normativo y la presencia del riesgo de litigio que tienen los directivos y las firmas de auditoría sobre su responsabilidad en la información financiera que emiten a los mercados de capitales.

En las distintas mediciones se incluye una serie de variables de control, con el objeto de poder atribuir los cambios en la calidad contable a los elementos del sistema (incluida las IFRS) en lugar de atribuirlo a cambios en los incentivos de la dirección; estos controles están relacionados con las decisiones contables voluntarias tomadas por la directivos (Barth et al., 2008) y con las características del entorno económico (el país y la industria) en el que opera cada empresa, los cuales podrían tener influencia en las magnitudes contables.

Las expresiones de la calidad contable de las empresas que adoptaron los estándares IFRS, son comparadas en sus periodos pre y post-adopción bajo un estudio de corte transversal (cross-sectional), siendo la misma empresa su propio control para garantizar la constancia de las características económicas de la misma y así los cambios en la calidad contable puedan ser atribuidos a la aplicación de dichos estándares.

Para controlar los efectos del entorno económico sobre las magnitudes contables, se ha incluido variables independientes como la industria, el país y el año de información analizado, de esta manera se capturan los efectos del entorno económico de modo que no puedan alterar los efectos realmente atribuidos al sistema contable, además las empresas de la muestra adoptaron los estándares IFRS en distintos periodos de tiempo y esto mitiga el efecto potencial del cambio en el ambiente económico.

Para evaluar la significatividad estadística de las diferencias en cada una de las expresiones de un periodo a otro, se utiliza un t-test basado en la distribución empírica de las diferencias entre periodos; se emplea el método de re-muestreo (bootstrapping) para la obtención de la distribución empírica de las diferencias, en el que aleatoriamente se selecciona con reemplazo desde cada sub-muestra original (GAAP locales e IFRS), el mismo número de observaciones de cada una de ellas para conformar una muestra aleatoria de igual tamaño que las originales, luego se calcula cada expresión de la calidad contable en dicha muestra aleatoria y se halla la diferencia entre los dos periodos analizados. Este procedimiento se repite 1000 veces y así se obtiene la distribución empírica de las diferencias en cada una de las expresiones. Este procedimiento se extiende para todas las expresiones, excepto en aquellas en las que se evalúa la significatividad a través del P-Value de los coeficientes respectivos de alguna de las variables. 


\section{Manipulación del resultado (atributos contables)}

Siguiendo a Barth et al. (2008), se utilizan cuatro (4) indicadores para la medición de la manipulación del resultado, y con estos se prueba la primera de las hipótesis definida (H1), tres (3) de los indicadores están relacionados con el alisamiento del resultado y el restante indicador, hace referencia a la manipulación del resultado hacia un objetivo; Barth et al. (2008), han documentado que a menor alisamiento del resultado y a menor manipulación del resultado hacia un objetivo, menor es la manipulación del mismo y por tanto mayor será la calidad contable.

\section{Alisamiento del resultado}

Los indicadores de medición utilizados son: la varianza del cambio en el resultado contable $(\Delta \mathrm{NI})$ (Lang et al., 2006), el ratio de la varianza del cambio en el resultado contable sobre la varianza del cambio en el Cash flow operativo $(\Delta \mathrm{NI} / \Delta \mathrm{CFO}$ ) (Lang et al., 2003) y la correlación de Spearman entre los ajustes por devengo (ACCRUALS o ACC) y el Cash flow operativo CFO, Corr (ACC, CFO) (Leuz et al., 2003).

De todos ellos se toman los residuos obtenidos de su regresión respectiva definidos de la siguiente manera: $\Delta \mathrm{NI}^{*},(\Delta \mathrm{NI} * / \mathbf{\Delta C F O} *), \operatorname{corr}\left(\mathrm{ACC}^{*}, \mathrm{CFO} *\right)$; la decisión de tomar los residuos obedece a que cada indicador es sensible a una serie de factores no relacionados con el sistema contable en general, que han quedado recogidos en los controles definidos para su regresión respectiva; se espera que los residuos de cada regresión correspondan a aquellas variables no contenidas en las variables de control, las cuales explicarían parte de los cambios en los indicadores definidos y se refieren a los elementos del sistema contable.

Los indicadores se interpretan como sigue:

$(\Delta \mathrm{NI})$ : ante una pequeña varianza en los residuos del cambio en el resultado contable, debería ser evidencia de la existencia de alisamiento del resultado (Barth et al., 2008).

$(\triangle \mathrm{NI} * / \triangle \mathrm{CFO} *)$ : ante un ratio menor en el periodo de aplicación de IFRS respecto al ratio en el periodo de GAAP locales, es evidencia de la existencia de alisamiento del resultado; investigaciones previas documentan que las empresas con mayor volatilidad en su cash flow operativo, típicamente presentan una mayor volatilidad en el resultado contable. Por tanto, si las empresas alisan el resultado, se espera que la varianza en los residuos del cambio en el resultado contable, sea más baja que la varianza en los residuos del cambio en el Cash flow operativo, y esto se traduce en un menor ratio entre las mismas (Lang et al., 2003).

Corr (ACC*, CFO*): una mayor correlación negativa entre el CFO y los ajustes por devengo es indicativo de mayor alisamiento del resultado (manipulación del resultado), lo 
anterior sugiere que las empresas utilizan los ajustes por devengo para alisar la variabilidad en los flujos de caja (Land \& Lang, 2002; Lang et al., 2003).

El $\Delta \mathrm{NI}^{*}$ son los residuos de la siguiente ecuación:

$$
\begin{aligned}
\Delta \mathrm{NI}_{i t}=\quad & \alpha+\beta_{1} \text { SIZE }_{i t}+\beta_{2} \text { GROWTH }_{i t}+\beta_{3} \text { EISSUE }_{i t}+\beta_{4} \text { LEV }_{i t}+\beta_{5} \text { DISSUE }_{i t} \\
& +\beta_{6} \text { TURN }_{i t}+\beta_{7} \text { CFO }_{i t}+\beta_{8} \text { AUD } \\
\text { it } & +\beta_{9} \text { NUMEX }_{i t}+\beta_{10} \text { XLIST }_{i t} \\
& +\sum_{l=1}^{5} \beta_{l+10} \text { YEAR }_{i}+\sum_{l=1}^{19} \beta_{l+15} \text { Country }_{i} \\
& +\sum_{m=1}^{18} \beta_{m+34} \text { Industry }_{i}+\varepsilon_{i t}
\end{aligned}
$$

Donde:

Para la empresa $i$ en el año $t$,

$\Delta \mathrm{NI}=$ cambio en la utilidad neta antes de partidas extraordinarias dividido por el total de activos al final del año.

SIZE= logaritmo natural del total de activos al final del año (tamaño de la compañía).

GROWTH= cambio porcentual en las ventas.

EISSUE $=$ cambio porcentual en el capital contable.

$\mathrm{LEV}=$ pasivos totales dividido por el valor en libros del patrimonio, ambos al final del periodo (nivel de apalancamiento).

DISSUE $=$ cambio porcentual en los pasivos totales.

TURN= ventas dividido por el total de activos al final del año (rotación de los activos).

$\mathrm{CFO}=$ Cash Flow operativo dividido por el total de activos, ambos al final del año.

$\mathrm{AUD}=$ variable dicótoma, toma valores de uno (1) si las empresas son auditadas por alguna Big4 (PWC, KPMG, EY, y DELOITTE) y cero (0) en otro caso.

NUMEX = número de bolsas en las cuales cotiza las acciones de la empresa.

XLIST = variable dicótoma, toma valores de uno (1) si la empresa cotiza en algún mercado accionario de los Estados Unidos y dicho mercado no es su bolsa principal.

YEAR $=$ año de reporte de información.

Country= indicador variable, siendo Argentina usada como referencia (Benchmark). El total de países en la muestra es de 20, para efectos del modelo econométrico se establecen 19 variables, para evitar el problema de la multicolinealidad perfecta.

Industry $=$ indicador variable de clasificación de la industria, a partir del código NACE proporcionado por la base de datos de información financiera ORBIS, siendo la Agricultura 
usada como referencia (Benchmark). El total de industrias en la muestra es 19, para efectos del modelo econométrico se establecen 18 variables para evitar el problema de la multicolinealidad perfecta.

El $\triangle \mathrm{CFO}^{*}$ son los residuos de la siguiente ecuación:

$$
\begin{aligned}
\Delta C F O_{i t}=\quad & \alpha+\beta_{1} \text { SIZE }_{i t}+\beta_{2} \text { GROWTH }_{i t}+\beta_{3} \text { EISSUE }_{i t}+\beta_{4} L E V_{i t}+\beta_{5} \text { DISSUE }_{i t} \\
& +\beta_{6} \text { TURN }_{i t}+\beta_{7} \text { CFO }_{i t}+\beta_{8} A U D_{i t}+\beta_{9} \text { NUMEX }_{i t}+\beta_{10} \text { XLIST }_{i t} \\
& +\sum_{l=1}^{5} \beta_{l+10} \text { YEAR }_{i}+\sum_{l=1}^{19} \beta_{l+15} \text { Country }_{i} \\
& +\sum_{m=1}^{18} \beta_{m+34} \text { Industry }_{i}+\varepsilon_{i t}
\end{aligned}
$$

Donde:

$\triangle \mathrm{CFO}=$ cambio en el flujo de caja operativo dividido por el total de activos al final del año. Las demás variables fueron definidas arriba.

Por tanto, el segundo indicador se basa en el ratio de los residuos obtenidos desde la ecuación 1 y $2,\left(\Delta \mathrm{NI} * / \Delta \mathrm{CFO}^{*}\right)$, comparando dicho ratio entre los periodos de GAAP locales e IFRS.

$\mathrm{CFO}^{*}$ y $\mathrm{ACC}^{*}$ son los residuos de las siguientes ecuaciones:

$$
\begin{aligned}
\text { CFO }_{i t}= & \alpha+\beta_{1} \text { SIZE }_{i t}+\beta_{2} \text { GROWTH }_{i t}+\beta_{3} \text { EISSUE }_{i t}+\beta_{4} \text { LEV }_{i t}+\beta_{5} \text { DISSUE }_{i t} \\
& +\beta_{6} \text { TURN }_{i t}+\beta_{7} \text { AUD }_{i t}+\beta_{8} \text { NUMEX }_{i t}+\beta_{9} \text { XLIST }_{i t}+\sum_{l=1}^{5} \beta_{l+9} \text { YEAR }_{i} \\
& +\sum_{l=1}^{19} \beta_{l+14} \text { Country }_{i}+\sum_{m=1}^{18} \beta_{m+33} \text { Industry }_{i}+\varepsilon_{i t} \\
\text { ACC }_{i t}=\quad \alpha+ & \beta_{1} \text { SIZE E }_{i t}+\beta_{2} \text { GROWTH }_{i t}+\beta_{3} \text { EISSUE }_{i t}+\beta_{4} \text { LEV }_{i t}+\beta_{5} \text { DISSUE }_{i t} \\
& +\beta_{6} \text { TURN }_{i t}+\beta_{7} \text { AUD }_{i t}+\beta_{8} \text { NUMEX }_{i t}+\beta_{9} \text { XLIST }_{i t}+\sum_{l=1}^{5} \beta_{l+9} Y E A R_{i} \\
& +\sum_{l=1}^{19} \beta_{l+14} \text { Country }_{i}+\sum_{m=1}^{18} \beta_{m+33} \text { Industry }_{i}+\varepsilon_{i t}
\end{aligned}
$$


Donde:

$\mathrm{CFO}=$ es el flujo de caja operativo dividido por el total de activos al final del año.

$\mathrm{ACC}=$ son los ajustes por devengo dividido por el total de activos al final del año. El total de ajustes por devengo es obtenido del resultado contable una vez sustraído el cash flow operativo $(\mathrm{ACC}=\mathrm{NI}-\mathrm{CFO})$.

Las demás variables fueron definidas arriba.

Se obtiene la correlación de los residuos desde las ecuaciones 3 y $4 \mathrm{CFO}^{*}$ y ACC* y se compara para ambos periodos.

En ambas ecuaciones se excluye la variable independiente CFO.

Desde las ecuaciones 1 a la 4 se estima las regresiones separando las observaciones para el periodo de GAAP locales y el periodo IFRS.

\section{Manipulación del resultado hacia un objetivo}

Se operacionaliza esta expresión a través de la frecuencia de reporte de beneficios mínimos y se evidencia con el coeficiente SPOS (Small Positive Net Income) contenido en la regresión (5) (Burgstahler \& Dichev, 1997), se obtiene un único coeficiente para ambos periodos y con éste, se examina si en un periodo con respecto al otro existe mayor probabilidad de manipulación de ganancias hacia resultados mínimos positivos.

Los resultados de este modelo son comprobados a través de la regresión bajo el modelo LOGIT y se agrupan todas las observaciones de ambos periodos.

$$
\begin{aligned}
\operatorname{Period}(0,1)_{i t} & =\alpha+\beta_{1} \text { SIZE }_{i t}+\beta_{2} \text { GROWTH }_{i t}+\beta_{3} \text { EISSUE }_{i t}+\beta_{4} L E V_{i t}+\beta_{5} \text { DISSUE }_{i t} \\
& +\beta_{6} \text { TURN }_{i t}+\beta_{7} \text { CFO }_{i t}+\beta_{8} A U D_{i t}+\beta_{9} N U M E X_{i t}+\beta_{10} X_{L I S T} \\
& +\beta_{11} \text { SPOS }_{i t}+\varepsilon_{i t}
\end{aligned}
$$

Donde:

Period $(0,1)=$ es una variable dicótoma, toma valores de uno (1) para las observaciones pertenecientes al periodo de IFRS y cero (0) en otro caso.

SPOS= toma valores de uno (1) si el resultado contable escalado por el total de activos al final del año se encuentra entre 0 y 0.01 y cero (0) en otro caso (Lang et al., 2003). Un coeficiente negativo de la variable SPOS indica que la empresa manipula el resultado hacia beneficios mínimos con mayor frecuencia en el periodo de GAAP locales que en el periodo IFRS, lo que se traduce en menor manipulación del resultado para este último periodo.

Las demás variables fueron definidas arriba. 


\section{Reconocimiento oportuno de pérdidas (atributos contables)}

El reconocimiento oportuno de pérdidas opera bajo la presunción que los estándares IFRS permiten políticas contables direccionadas a reconocer, en forma oportuna, las pérdidas en el resultado reportado, y esto a su vez es indicio de un mejoramiento en la calidad contable.

Esta expresión se operacionaliza a través de la frecuencia al reconocer grandes pérdidas (Ball, 2001) y con ésta, se prueba la segunda de las hipótesis (H2).

$$
\begin{aligned}
\operatorname{Period}(0,1)_{i t} & =\alpha+\beta_{1} \text { SIZE }_{i t}+\beta_{2} \text { GROWTH }_{i t}+\beta_{3} \text { EISSUE }_{i t}+\beta_{4} L_{E V_{i t}}+\beta_{5} \text { DISSUE }_{i t} \\
& +\beta_{6} \text { TURN }_{i t}+\beta_{7} \text { CFO }_{i t}+\beta_{8} \text { AUD }_{i t}+\beta_{9} \text { NUMEX }_{i t}+\beta_{10} \text { XLIST }_{i t} \\
& +\beta_{11} L N E G_{i t}+\varepsilon_{i t}
\end{aligned}
$$

Donde:

LNEG (Large negative Net Income)= es una variable dicótoma, toma valores de uno (1) para las observaciones en las cuales el resultado contable escalado por el total de activos al final del año es menor que -0.20 y cero ( 0 ) en otro caso. Un coeficiente positivo de LNEG indica que las empresas reconocen grandes pérdidas con mayor frecuencia en el periodo IFRS que en el de aplicación de GAAP locales, lo que es indicio de mayor calidad contable.

Las demás variables fueron definidas arriba.

Los resultados de este modelo son comprobados a través de la regresión bajo el modelo LOGIT y se agrupan todas las observaciones de ambos periodos.

\section{Relevancia valorativa (atributos de mercado)}

Se operacionaliza a través de tres indicadores o modelos de medición: Ohlson (1995), Bartov et al. (2005) y Basu (1997). Dichos modelos basan su análisis en la capacidad informativa que tienen las magnitudes contables en los mercados de capitales, permitiendo con ellos, probar la tercera hipótesis definida (H3).

El modelo de Ohlson (1995), se refiere al poder de explicación que tienen el resultado contable por acción (EPS) y valor en libros del patrimonio por acción (BVEPS) sobre los cambios en el precio de la acción (P); además de incluir variables de control que tienen efectos fijos sobre el precio como lo son la industria, el país y el año de reporte de información. Dentro de la ecuación se agrega la variable independiente POST, y su interacción con las variables EPS y BVEPS. El término POST es una variable dicótoma, toma valores de uno (1) para el periodo IFRS y cero (0) en otro caso. Las diferencias en la relevancia de valor entre 
los periodos, se espera sea reflejado en el coeficiente significativamente positivo entre los términos de interacción con la variable POST, en $\beta_{4}$ y $\beta_{5}$ de la ecuación (7).

Teniendo en cuenta la periocidad en que la información contable esté disponible públicamente, el precio de la acción se mide 6 meses después de finalizar el periodo contable anual (Lang et al., 2003; Lang et al., 2006).

$$
\begin{aligned}
& P_{i t}=\alpha+\beta_{1} \text { BVEPS }_{i t}+\beta_{2} \text { EPS }_{i t}+\beta_{3} \text { POST }_{i t}+\beta_{4} \text { BVEPS }_{i t} x P O S T_{i t}+\beta_{5} E P S_{i t} x P O S T_{i t} \\
&+\sum_{l=1}^{12} \beta_{l+5} \text { Country }_{i} \\
&+\sum_{m=1}^{17} \beta_{m+17} \text { Industry }_{i}+\sum_{m=1}^{8} \beta_{m+34} Y E A R_{i}+\varepsilon_{i t}
\end{aligned}
$$

En el modelo de Bartov et al. (2005), se espera que el anuncio del resultado contable afecte el precio de la acción y por tanto la rentabilidad de la misma, la variable dependiente en la ecuación es la rentabilidad (RETURN) mientras que las variables independientes son EPS, POST y su respectivo término de interacción, además de agregar las variables industria, país y año de reporte de información. La diferencia entre la pertinencia de valor para los dos periodos analizados, se espera sea reflejada en el coeficiente significativamente positivo de la interacción entre POST y EPS, $\beta_{3}$ de la ecuación (8). La Rentabilidad (RETURN) es calculada como el logaritmo natural del ratio del precio de la acción 6 meses después de finalizar el año fiscal sobre el precio de la acción 6 meses antes de finalizar el mismo, ajustado por los dividendos (Lang et al., 2006).

$$
\begin{aligned}
\text { RETURN } *_{i t}= & \alpha+\beta_{1} \text { EPS }_{i t}+\beta_{2} \text { POST }_{i t}+\beta_{3} \text { EPS }_{i t} x P O S T_{i t}+\sum_{l=1}^{12} \beta_{l+3} \text { Country }_{i} \\
& +\sum_{m=1}^{17} \beta_{m+15} \text { Industry }_{i}+\sum_{m=1}^{8} \beta_{m+32} \text { YEAR }_{i}+\varepsilon_{i t}
\end{aligned}
$$

El modelo propuesto por Basu (1997), considera el resultado contable en función de las rentabilidades, incorporando dentro del modelo, la variable BAD y su interacción con la variable rentabilidad; la variable BAD toma valores de uno (1) en los periodos en que las firmas reportan rentabilidades negativas y cero (0) en otro caso; se parte de la presunción 
que el resultado contable reportado se ve finalmente reflejado en el precio de la acción, la cuestión por valorar es si las noticias, especialmente las malas noticias, son incorporadas en el resultado contable de manera oportuna y esto se confirma a través del coeficiente de interacción entre las rentabilidades y la variable de malas noticias.

Se incorpora además, la variable POST y su interacción con las demás variables indicadas hasta ahora para este modelo. Partiendo de la presunción que las IFRS contienen políticas contables que permiten incorporar oportunamente las pérdidas en el resultado (mayor rapidez), el coeficiente estimado de la ecuación (9) resultará ser positivo y significativo. La magnitud del coeficiente, es una medida de la rapidez o sensibilidad incremental con que las malas noticias son reflejadas en el resultado contable respecto a las buenas noticias durante el periodo de aplicación de IFRS (conservadurismo contable).

$$
\begin{aligned}
(E P S / P)_{i t}= & \alpha+\beta_{1} \text { RETURN }_{i t}+\beta_{2} \text { BAD }_{i t}+\beta_{3} \text { RETURN }_{i t} x B A D_{i t}+\beta_{4} \text { POST }_{i t} \\
& +\beta_{5} \text { RETURN }_{i t} x \text { OSST }_{i t}+\beta_{6} \text { BAD }_{i t} x \text { OOST }_{i t} \\
& +\beta_{7} \text { RETURN }_{i t} x B A D_{i t} x \text { POST }_{i t}+\sum_{l=1}^{12} \beta_{l+7} \text { Country }_{i} \\
& +\sum_{m=1}^{17} \beta_{m+19} \text { Industry }_{i}+\sum_{m=1}^{8} \beta_{m+36} \text { YEAR }_{i}+\varepsilon_{i t}
\end{aligned}
$$

Donde:

$(\mathrm{EPS} / \mathrm{P})=$ es el resultado por acción deflactado por el precio de la acción al inicio del periodo.

Las demás variables fueron definidas arriba.

\section{Muestra, Distribución y estadísticos descriptivos}

\section{Selección y distribución de la muestra}

La Tabla 1 presenta la selección de la muestra inicial (Panel A) a partir de 5.545 empresas reportadas como activas en la base de datos de información financiera ORBIS, listadas durante el periodo de análisis 2006 a 2016, con al menos un reporte anual bajo IFRS y domiciliadas en Latinoamérica o el Caribe (41.602 observaciones en total); son excluidas 3.178 compañías, 1.820 por ser listadas en mercados de valores distintos a los latinos o del Caribe (14.786 observaciones) y 1.358 empresas sin reporte de estados financieros bajo principios contables 
locales (9.086 observaciones), conformándose una muestra inicial por 2.367 compañías y permaneciendo un total de 17.730 observaciones.

En el Panel B (Panel C) de la misma tabla, se reporta la conformación de la muestra para las pruebas relacionadas con los atributos contables (atributos de mercado) partiendo de las observaciones seleccionadas en la muestra inicial; en el Panel B (Panel C) se eliminan 13.159 (15.163) observaciones, 5.209 (14.384) por datos perdidos, 7.099 (364) por observaciones que tan sólo presentan información financiera bajo uno de los dos regímenes contables y las restantes 851 (415) observaciones dado que pertenecen al periodo de transición desde el marco normativo local a IFRS, conformándose una muestra final para el Panel B (Panel C) por 734 (379) compañías y permaneciendo un total de 4.571 (2.567) observaciones.

Tabla 1

Selección de la muestra

\begin{tabular}{lllll}
\hline & $\begin{array}{l}\text { Núm. em- } \\
\text { presas }\end{array}$ & $\begin{array}{l}\text { Núm. Obs. } \\
\text { GAAP Locales }\end{array}$ & IFRS & Total \\
\hline Panel A: Muestra inicial & & & & \\
\hline $\begin{array}{l}\text { Empresas clasificadas como activas en ORBIS, } \\
\text { cotizadas o que cotizaron durante el periodo }\end{array}$ & & 14494 & & \\
$\begin{array}{l}\text { 2006 a 2016, con al menos tres años de in- } \\
\text { formación financiera disponible, al menos } \\
\text { un reporte anual bajo IFRS y domiciliadas en }\end{array}$ & & & \\
$\begin{array}{l}\text { América Latina o el Caribe. } \\
\text { Empresas no listadas en los mercados latinos } \\
\text { o del Caribe. }\end{array}$ & $(1820)$ & $(339)$ & $(14447)$ & \\
$\begin{array}{l}\text { Empresas sin reporte de información financie- } \\
\text { ra bajo principios contables locales (GAAP }\end{array}$ & $(1358)$ & $(2799)$ & \\
Locales). & & & \\
\hline Total Muestra inicial & 2367 & 11356 & \\
\hline
\end{tabular}

Panel B: Muestra para el test de los atributos contables

\begin{tabular}{|c|c|c|c|c|}
\hline Muestra desde el Panel A & 2367 & 11356 & 6374 & 17730 \\
\hline $\begin{array}{l}\text { Datos perdidos para las variables relacionadas } \\
\text { con la manipulación del resultado y el reco- } \\
\text { nocimiento oportuno de pérdidas. }\end{array}$ & & (3 325) & $(1884)$ & $(5209)$ \\
\hline $\begin{array}{l}\text { Empresas que reportan observaciones en sólo } \\
\text { un régimen contable. }\end{array}$ & & (6 333) & $(766)$ & (7 099) \\
\hline $\begin{array}{l}\text { Observaciones pertenecientes al periodo de } \\
\text { transición de GAAP Locales a IFRS. }\end{array}$ & & $(108)$ & $(743)$ & $(851)$ \\
\hline Total Muestra Panel B & 734 & 1590 & 2981 & 4571 \\
\hline
\end{tabular}


Panel C: Muestra para el test de los atributos de mercado

\begin{tabular}{lllll}
\hline Muestra desde el Panel A & 2367 & 11356 & 6374 & 17730 \\
\hline $\begin{array}{l}\text { Datos perdidos para las variables relacionadas } \\
\text { con la relevancia valorativa. }\end{array}$ & $(10285)$ & $(4099)$ & $(14384)$ \\
$\begin{array}{l}\text { Empresas que reportan observaciones en sólo } \\
\text { un régimen contable. }\end{array}$ & $(57)$ & $(307)$ & $(364)$ \\
$\begin{array}{l}\text { Observaciones pertenecientes al periodo de } \\
\text { transición de GAAP Locales a IFRS. }\end{array}$ & $(45)$ & $(370)$ & $(415)$ \\
\hline Total Muestra Panel C & 379 & 969 & 1598 & 2567 \\
\hline
\end{tabular}

Fuente: Elaboración propia

En la Tabla 2 se presenta la distribución de la muestra por país (Panel A), industria (Panel B) y año de análisis (Panel C) clasificando de acuerdo con el tipo de prueba: atributos contables (Muestra A) y atributos de mercado (Muestra B), en ambos tipos de muestra contenidos en el Panel A se observa una mayor participación de compañías y observaciones ubicadas en Brasil, seguido por observaciones pertenecientes a Chile, Argentina y Perú.

En el Panel B, para la muestra relacionada con los atributos contables (Muestra A) se observa una mayor participación de observaciones pertenecientes a la industria manufacturera y a la de suministro de energía, seguida de empresas pertenecientes a la industria de actividades financieras; las observaciones de empresas ubicadas en los sectores de comercio al por menor y por mayor, transporte y almacenamiento y agricultura y ganadería representan alta participación del total de las observaciones.

Para la muestra relacionada con los atributos de mercado (Muestra B), si bien las observaciones pertenecientes a los sectores manufacturero y de suministro de gas representan más de la mitad del total de la muestra, las industrias comercial, constructora, extractiva y de actividades de comunicación tienen un papel representativo del total de la misma.

Atendiendo al Panel C, se observa una mayor participación de las observaciones entre los años 2007 a 2009 bajo el régimen GAAP Locales, con posterioridad a estos años se presenta una creciente participación de las observaciones bajo el régimen contable IFRS, siendo estos últimos años en los que la mayoría de los países latinoamericanos han incorporado a sus legislaciones la aplicación obligatoria de las IFRS. 
Tabla 2

Distribución de la muestra

\begin{tabular}{|c|c|c|c|c|c|c|}
\hline \multirow[t]{2}{*}{$\begin{array}{l}\text { País } \\
\text { Núm. Empresas }\end{array}$} & \multicolumn{3}{|c|}{ Muestra A (atributos contables) } & \multicolumn{2}{|c|}{$\begin{array}{l}\text { Muestra B (atributos } \\
\text { de mercado) }\end{array}$} & \\
\hline & Núm. Obs. $\%$ Obs. & \multicolumn{2}{|c|}{ Núm. Empresas } & \multirow[t]{2}{*}{ Núm. Obs. } & \multirow[t]{2}{*}{$\%$ Obs. } & \\
\hline Panel A-Distribución de & as y observaciones por & país & & & & \\
\hline Argentina & 86 & 539 & $11.8 \%$ & 46 & 333 & $13.0 \%$ \\
\hline Bahamas & 2 & 7 & $0.2 \%$ & 2 & 8 & $0.3 \%$ \\
\hline Bermudas & 8 & 43 & $0.9 \%$ & 6 & 43 & $1.7 \%$ \\
\hline Bolivia & 27 & 153 & $3.3 \%$ & 0 & 0 & $0.0 \%$ \\
\hline Brasil & 268 & 1732 & $37.9 \%$ & 181 & 1256 & $48.9 \%$ \\
\hline Chile & 171 & 1088 & $23.8 \%$ & 93 & 640 & $24.9 \%$ \\
\hline Colombia & 20 & 109 & $2.4 \%$ & 5 & 24 & $0.9 \%$ \\
\hline Costa Rica & 2 & 12 & $0.3 \%$ & 0 & 0 & $0.0 \%$ \\
\hline Ecuador & 32 & 181 & $4.0 \%$ & 0 & 0 & $0.0 \%$ \\
\hline El Salvador & 1 & 6 & $0.1 \%$ & 0 & 0 & $0.0 \%$ \\
\hline Honduras & 1 & 5 & $0.1 \%$ & 6 & 21 & $0.8 \%$ \\
\hline Islas Caimán & 2 & 7 & $0.2 \%$ & 3 & 17 & $0.7 \%$ \\
\hline Islas Vírgenes Británicas & 3 & 12 & $0.3 \%$ & 3 & 19 & $0.7 \%$ \\
\hline México & 3 & 8 & $0.2 \%$ & 1 & 2 & $0.1 \%$ \\
\hline Nicaragua & 1 & 5 & $0.1 \%$ & 0 & 0 & $0.0 \%$ \\
\hline Panamá & 3 & 16 & $0.4 \%$ & 1 & 8 & $0.3 \%$ \\
\hline Paraguay & 29 & 166 & $3.6 \%$ & 0 & 0 & $0.0 \%$ \\
\hline Perú & 57 & 383 & $8.4 \%$ & 25 & 156 & $6.1 \%$ \\
\hline Uruguay & 6 & 34 & $0.7 \%$ & 0 & 0 & $0.0 \%$ \\
\hline Venezuela & 12 & 65 & $1.4 \%$ & 7 & 40 & $1.6 \%$ \\
\hline Total & 734 & 4571 & $100 \%$ & 379 & 2567 & $100 \%$ \\
\hline
\end{tabular}

Industria

Núm. Empresas
Muestra A (atributos contables)

Muestra B (atributos de mer-

cado)

Núm.Obs. \% Obs. Núm. Empresas Núm. Obs. \% Obs.

Panel B - Distribución de las empresas y observaciones por industria

Agricultura, ganadería, silvicultura

y pesca

Industrias extractivas

Industria manufacturera
40

26

239
256

$5.6 \% \quad 18$

$160 \quad 3.5 \% \quad 18$

$1524 \quad 33.3 \% \quad 144$
104

$4,1 \%$

128

$995 \quad 38.8 \%$ 
Suministro de energía eléctrica, gas y vapor 104 $655 \quad 14.3 \% \quad 53$

Suministro de agua, actividades de

\begin{tabular}{lllllll} 
saneamiento & 17 & 106 & $2.3 \%$ & 5 & 38 & $1.5 \%$ \\
Construcción & 38 & 238 & $5.2 \%$ & 23 & 155 & $6.0 \%$ \\
Comercio al por mayor y al por menor & 51 & 308 & $6.7 \%$ & 24 & 160 & $6.2 \%$ \\
Transporte y almacenamiento & 41 & 262 & $5.7 \%$ & 15 & 108 & $4.2 \%$ \\
Hostelería & 7 & 46 & $1.0 \%$ & 1 & 8 & $0.3 \%$ \\
Information et communication & 28 & 167 & $3.7 \%$ & 19 & 132 & $5.1 \%$ \\
Actividades financieras y de seguros & 65 & 378 & $8.3 \%$ & 21 & 135 & $5.3 \%$ \\
Actividades inmobiliarias & 34 & 212 & $4.6 \%$ & 25 & 170 & $6.6 \%$ \\
Actividades profesionales, científicas & & & & & & \\
y técnicas & 9 & 53 & $1.2 \%$ & 3 & 18 & $0.7 \%$ \\
Actividades administrativas & 3 & 17 & $0.4 \%$ & 2 & 13 & $0.5 \%$ \\
Administración pública & 2 & 7 & $0.2 \%$ & 0 & 0 & $0.0 \%$ \\
Educación & 5 & 39 & $0.6 \%$ & 1 & 5 & $0.2 \%$ \\
Actividades sanitarias & 6 & $0.9 \%$ & 3 & 21 & $0.8 \%$ \\
Actividades artísticas y recreativas & 11 & 52 & $1.1 \%$ & 2 & 7 & $0.3 \%$ \\
Otros servicios & 8 & 4571 & $100 \%$ & 379 & 2567 & $100 \%$ \\
\hline Total & 734 & & & \\
\hline
\end{tabular}

Año análisis Muestra A (Atributos contables)

Muestra B (Atributos de mercado)

\begin{tabular}{lllll}
\hline GAAP Locales & IFRS & GAAP Locales & IFRS \\
\hline Núm.Obs. $\%$ Obs. & Núm. Obs. \% Obs. & Núm. \% Obs. & N ú m . \% Obs.
\end{tabular}

Obs.

Obs.

\begin{tabular}{lllllllll}
\hline \multicolumn{7}{l}{ Panel C - Distribución de las observaciones por año de análisis } \\
\hline 2007 & 0 & $0.0 \%$ & 0 & $0.0 \%$ & 248 & $25.6 \%$ & 0 & $0.0 \%$ \\
2008 & 499 & $31.4 \%$ & 0 & $0.0 \%$ & 322 & $33.2 \%$ & 0 & $0.0 \%$ \\
2009 & 520 & $32.7 \%$ & 0 & $0.0 \%$ & 250 & $25.8 \%$ & 4 & $0.3 \%$ \\
2010 & 222 & $14.0 \%$ & 140 & $4.7 \%$ & 69 & $7.1 \%$ & 98 & $6.1 \%$ \\
2011 & 191 & $12.0 \%$ & 438 & $14.7 \%$ & 57 & $5.9 \%$ & 268 & $16.8 \%$ \\
2012 & 141 & $8.9 \%$ & 480 & $16.1 \%$ & 23 & $2.4 \%$ & 279 & $17.5 \%$ \\
2013 & 17 & $1.1 \%$ & 553 & $18.6 \%$ & 0 & $0.0 \%$ & 313 & $19.6 \%$ \\
2014 & 0 & $0.0 \%$ & 674 & $22.6 \%$ & 0 & $0.0 \%$ & 329 & $20.6 \%$ \\
2015 & 0 & $0.0 \%$ & 671 & $22.5 \%$ & 0 & $0.0 \%$ & 307 & $19.2 \%$ \\
2016 & 0 & $0.0 \%$ & 25 & $0.8 \%$ & 0 & $0.0 \%$ & 0 & $0.0 \%$ \\
\hline Total & 1590 & $100 \%$ & 2981 & $100 \%$ & 969 & $100 \%$ & 1598 & $100 \%$ \\
\hline
\end{tabular}

Fuente: Elaboración propia 


\section{Estadísticos descriptivos}

La Tabla 3 incluye los estadísticos descriptivos de las variables test y de control utilizadas en el análisis de la comparabilidad de la calidad contable; tal como se presenta en dicha tabla, existe una diferencia significativa en media (mediana), respecto al cambio del resultado contable $(\Delta \mathrm{NI})$ entre los periodos analizados, siendo mucho más bajo durante el periodo de aplicación de IFRS, al mismo tiempo, se observa una disminución significativa en media (mediana), en cuanto a la variación de los flujos de efectivos operacionales ( $\triangle \mathrm{CFO}$ ), además de un importe mucho más negativo y significativo en media y en mediana respecto a los ajustes por devengo, puede aludirse que parte de la disminución en la variación del resultado contable reportado no sólo obedece a variaciones negativas en los flujos de efectivo operativos, también a un mayor uso de los ajustes por devengo.

Respecto a la frecuencia en el reporte de grandes pérdidas (LNEG) se presenta un significativo incremento en media, durante el periodo de aplicación de IFRS, sugiriendo que posiblemente durante este último periodo se reconocen grandes pérdidas con mayor frecuencia respecto a las reconocidas durante los periodos de pre-adopción; referente a la frecuencia en el reporte de utilidades mínimas (SPOS), si bien en media presenta una disminución durante el periodo de aplicación de IFRS, no resulta tener significancia estadística, sugiriendo un mismo nivel de frecuencia al reportar utilidades mínimas entre ambos periodos.

Los estadísticos también indican sobre una disminución significativa en media (mediana) respecto al ratio del retorno de los activos (TURN) para el periodo de aplicación de IFRS, dicha disminución es explicada por una caída significativa en media (mediana) del crecimiento de las compañías, analizado a través de la variación en las ventas (GROWTH) y explicada además, por un crecimiento significativo en media (mediana) respecto al tamaño de las mismas medido a través de los activos (SIZE).

Se reporta una disminución significativa en las formas de financiación de la compañía (en media y en mediana) dado la variación en el patrimonio (EISSUE) y la variación en los pasivos (DISSUE), sin embargo, el nivel de apalancamiento (LEV) si bien registra un incremento en media (en mediana) para el periodo de aplicación de IFRS, sólo resulta ser significativo respecto a su mediana; los hallazgos anteriores no sugieren una relación entre el crecimiento de las compañías (SIZE) y la forma de financiación de las mismas; el aumento en el tamaño de las compañías durante el periodo IFRS, puede ser explicado por el impacto de la revaluación contenida en las IFRS, y ratificado con el mismo nivel en media y en mediana (sin significancia estadística) de los flujos de efectivo operativos de la entidad (CFO).

Los estadísticos también reportan que durante el periodo de aplicación de IFRS, las compañías sostienen un crecimiento significativo en cuanto al uso de servicios de auditoría por parte de las BIG4 (AUD) y en cuanto a la aceptación de su cotización en un mayor número 
de mercados de valores (NUMEX), sin embargo los resultados no sugieren cambio alguno respecto a la aceptación de su cotización en los mercados estadounidenses (XLIST).

Referente a las variables de medición de la relevancia valorativa, las IFRS impactan el importe del resultado contable (EPS) y del valor en libros del patrimonio por acción (BVEPS), disminuyendo significativamente (en media y mediana) al primero y aumentando significativamente (en mediana) el importe del patrimonio contable; el precio por acción (P) presenta una caída significativa en media (mediana) para el periodo de IFRS, siendo extensible a la rentabilidad por acción, aunque la disminución en ésta última no resulte ser estadísticamente significativa.

Tabla 3

Estadísticos Descriptivos: Comparación de variables bajo los periodos GAAP Locales e IFRS

\begin{tabular}{|c|c|c|c|c|c|}
\hline \multirow[b]{2}{*}{ Variable } & \multicolumn{3}{|c|}{ Periodo (GAAP Locales) } & \multicolumn{2}{|c|}{ Periodo (IFRS) } \\
\hline & $\begin{array}{l}\text { Igualdad en Va- } \\
\text { rianza }\end{array}$ & Media & Mediana & Media & Mediana \\
\hline Obs. & 1590 & & & 2981 & \\
\hline$\Delta \mathrm{NI}$ & $*$ & -0.001 & -0.002 & $-0.008 *$ & $-0.003 * *$ \\
\hline SIZE & $* * *$ & 11.971 & 12.035 & $12.764 *$ & $12.792 *$ \\
\hline GROWTH & $*$ & 0.173 & 0.111 & $-0.028^{*}$ & $-0.033^{*}$ \\
\hline EISSUE & $*$ & 0.123 & 0.082 & $-0.026^{*}$ & $-0.048^{*}$ \\
\hline LEV & $*$ & 1.421 & 0.970 & 1.465 & $1.010 * *$ \\
\hline DISSUE & $*$ & 0.172 & 0.100 & $-0.004 *$ & $-0.037 *$ \\
\hline TURN & $*$ & 0.717 & 0.630 & $0.620 *$ & $0.555^{*}$ \\
\hline SPOS & $* *$ & 0.074 & 0 & 0.067 & 0 \\
\hline LNEG & $*$ & 0.022 & 0 & $0.033^{* *}$ & $0 * *$ \\
\hline AUD & $*$ & 0.538 & 1 & $0.676^{*}$ & $1^{*}$ \\
\hline NUMEX & $* *$ & 0.955 & 1 & $1.065^{*}$ & $1^{*}$ \\
\hline XLIST & & 0.017 & 0 & 0.016 & 0 \\
\hline $\mathrm{CFO}$ & & 0.062 & 0.057 & 0.058 & 0.059 \\
\hline$\triangle \mathrm{CFO}$ & $*$ & 0.031 & 0.017 & $-0.004 *$ & $0.001 *$ \\
\hline ACC & $*$ & -0.015 & -0.012 & $-0.028 *$ & $-0.022 *$ \\
\hline Obs. & 969 & & & 1598 & \\
\hline EPS & & 0.379 & 0.160 & $0.119^{*}$ & $0.137 * *$ \\
\hline BVEPS & & 6.788 & 1.840 & 7.254 & $2.663 * * *$ \\
\hline P (PRECIO) & $*$ & 7.762 & 3.029 & $6.208^{*}$ & $2.546^{*}$ \\
\hline RETURN & $*$ & 4.891 & 4.854 & 4.780 & 4.729 \\
\hline
\end{tabular}

Fuente: Elaboración propia 
$*, * *, * * *$ Indica diferencia estadísticamente significativa entre periodos con un nivel $p<0.01, p<0.05, p<0.1$ respectivamente (prueba de dos colas), para la medición de significancia entre las medianas de las variables AUD, LNEG, SPOS se utiliza un test de comparación de proporciones basado en el test exacto de Fisher, para las demás variables se utiliza el test de suma de rangos de Mann-Whitney-Wilcoxon.

A pesar que el supuesto de normalidad en las perturbaciones no es confirmado con el test de Shapiro-Wilk, este supuesto se relaja debido a que el tamaño de la muestra es lo suficientemente grande (superior a 30 observaciones), por tanto se asume normalidad de acuerdo con el Teorema Central del Límite.

Se utiliza un t-test para la comparación de medias entre variables, excepto para aquellas variables en las que previamente su comparación de varianzas resulta ser diferente de cero (significativas), en estos casos se utiliza un t-test de comparación de medias con varianzas distintas.

En todas las variables de las regresiones, exceptuando las variables dicótomas, se controla los efectos de los valores outliers (winsorized) en los percentiles 5 y 95 .

\section{Resultados}

Los resultados comparativos de la calidad contable entre los periodos pre- y post-adopción son presentados en las Tablas 4 y 5 . La Tabla 4 presenta los hallazgos de la calidad contable incluyendo todas las observaciones disponibles agrupadas entre los periodos de análisis (LOCAL GAAP e IFRS), mientras que la Tabla 5, reporta los análisis comparativos únicamente entre el periodo inmediatamente anterior a la adopción de IFRS y el periodo inmediatamente posterior a la misma (muestra restringida).

El panel A de la Tabla 4, compara las expresiones relacionadas con el alisamiento del resultado, la primera de ellas, la variabilidad del cambio en el resultado contable $(\Delta \mathrm{NI} *)$ presenta una significativa disminución en el periodo de post-adopción respecto al periodo de pre-adopción ( 0.0020 vs. 0.0022 ), siendo contrario al coeficiente esperado, esto posiblemente sugiere un incremento en el alisamiento del resultado. Debido a que la variabilidad del resultado contable podría ser afectada por la variabilidad en los flujos de efectivo ( $\Delta \mathrm{CFO} *)$, y éstos últimos no son técnicas de discrecionalidad contable, se analiza el ratio de la variabilidad $(\triangle \mathrm{NI} *)$ / la variabilidad $(\triangle \mathrm{CFO} *)$, dicho ratio resulta tener un aumento estadísticamente significativo durante el periodo de post-adopción (0.2464 vs. 0.1992), lo que sugiere un aumento en la calidad contable para este último periodo.

Los hallazgos documentados hasta ahora para América Latina y el Caribe resultan ser contradictorios; Lin et al. (2012), presentan la misma situación para el caso alemán y sugieren que las medidas más concluyentes, serían aquellas que ajustan la volatilidad del resultado contable a la volatilidad del cash flow; de acuerdo con esto, la siguiente expresión analizada, la Correlación de $\mathrm{ACC}^{*}$ y CFO*, resulta ser menos negativa y estadísticamente significativa durante el periodo de post-adopción (-0.6702 vs. -0.7144); por tanto la evidencia disponible indica que durante el periodo de aplicación de IFRS, las empresas presentan un menor nivel de alisamiento del resultado (menor manipulación del resultado), lo que se traduce en mayor calidad contable. 
Respecto a la frecuencia en el reporte de utilidades mínimas (Panel B) y a la frecuencia en el reporte de grandes pérdidas (Panel C), los hallazgos no sugieren un cambio significativo entre periodos $(\mathrm{SPOS}=-0.1818)$ y $(\mathrm{LNEG}=0.2807)$ respectivamente; de acuerdo con esto, los resultados obtenidos en el Panel $\mathrm{C}$ permiten rechazar la segunda hipótesis.

Bajo el modelo propuesto por Ohlson (1995) contenido en el Panel D, presenta un coeficiente positivo y significativo para la magnitud incremental del valor en libros del patrimonio $\left(\beta_{4}=0.001\right)$ y negativo y significativo para la magnitud incremental del resultado contable durante el periodo de aplicación de IFRS $\left(\beta_{5}=-0.019\right)$, la disminución en la capacidad informativa del resultado contable es corroborado nuevamente a través del modelo propuesto por Bartov et al. (2005) $\left(\beta_{3}=-0.001\right)$; en general los resultados sugieren mayor (menor) capacidad explicativa del valor en libros del patrimonio (resultado contable) ante las variaciones en el precio por acción (precio y rentabilidad por acción), respecto al periodo de aplicación de estándares locales.

Finalmente, bajo el modelo propuesto por Basu (1997), el coeficiente de malas noticias resulta ser negativo sin significancia estadística durante el periodo de post-adopción $\left(\beta_{7}=-\right.$ 0.036) y positivo y significativo durante los periodos previos a la aplicación de las IFRS ( $\beta_{3}=0.032$ ); estos resultados indican que durante el periodo de aplicación de IFRS (LOCAL GAAP), las rentabilidades negativas se incorporan con igual (mayor) oportunidad en el resultado contable respecto a las buenas noticias, siendo indicativo de menor relevancia valorativa durante el periodo de post-adopción.

En general, los hallazgos obtenidos para Latinoamérica y el Caribe evidencian, respecto a la manipulación del resultado (atributos contables), un aumento en el nivel de la calidad contable durante el periodo de aplicación de IFRS, lo cual confirma la primera hipótesis definida; sin embargo, bajo las métricas relacionadas con la relevancia valorativa (atributos de mercado), los hallazgos sugieren una disminución en la capacidad informativa de las magnitudes contables en los mercados de capitales, rechazando así la tercera hipótesis. Los anteriores resultados son similares a los obtenidos para la Unión Europea (Zeghal et al., 2012), en los que a pesar de evidenciarse un aumento en la calidad contable vía disminución en la manipulación del resultado, la información contable preparada bajo IFRS no es apreciada con mayor utilidad por parte de los inversores.

Los resultados documentados hasta ahora, son sustentados a su vez por Cahan et al. (2009), quienes declaran que, así el sistema de contabilidad bajo IFRS genere información contable de alta calidad en un país con una débil infraestructura de protección al inversor, la información seguirá siendo menos importante para los participantes en el mercado.

Iatridis et al. (2010), evidencian en su análisis que a partir del segundo periodo de aplicación de IFRS, las empresas reportan una disminución en el nivel de manipulación del resultado y un incremento en la relevancia valorativa de la información financiera en los mercados de 
capitales de Grecia, soportado en la presunción de mayor claridad y familiarización con el proceso de implementación y uso de las IFRS; de acuerdo con lo anterior, a continuación se incluye un análisis adicional (Test de Robustez) de la comparabilidad de la calidad contable solamente considerando el periodo previo y el periodo posterior a la aplicación de las IFRS (muestra restringida) (Tabla 5).

Tabla 4

Comparación de la calidad contable (todas las observaciones)

\begin{tabular}{|c|c|c|c|c|c|c|c|}
\hline & \multicolumn{3}{|l|}{ Obs. } & \multirow{2}{*}{$\begin{array}{l}\text { Pre-adop- } \\
\text { ción } \\
\text { (LOCAL } \\
\text { GAAP) } \\
\end{array}$} & \multirow[t]{2}{*}{$\begin{array}{l}\text { Post-adopción } \\
\text { (IFRS) }\end{array}$} & \multirow[t]{2}{*}{$\begin{array}{l}\text { Signo } \\
\text { Esperado }\end{array}$} & \multirow[t]{2}{*}{$\begin{array}{r}\text { Diferencia } \\
\text { (Post-Pre) }\end{array}$} \\
\hline & Pre & \multicolumn{2}{|l|}{ Post } & & & & \\
\hline \multicolumn{8}{|c|}{ Panel A-Alisamiento del Resultado } \\
\hline $\begin{array}{l}\text { Varianza } \\
\text { del } \Delta \mathrm{NI}^{*}\end{array}$ & 1590 & & 2981 & 0.0022 & 0.0020 & + & $-0.0002 * * *$ \\
\hline $\begin{array}{l}\text { Varianza } \\
\operatorname{del} \Delta \mathrm{NI} * / \Delta \mathrm{CFO} *\end{array}$ & 1590 & & 2981 & 0.1992 & 0.2464 & + & $0.0471 *$ \\
\hline $\begin{array}{l}\text { Correlación de } \\
\mathrm{ACC}^{*} \mathrm{yCFO}^{*}\end{array}$ & 1590 & & 2981 & -0.7144 & -0.6702 & + & $0.0441 * *$ \\
\hline \multicolumn{8}{|c|}{ Panel B - Manipulación hacia un Resultado Objetivo } \\
\hline & 4571 & & -0.1818 & & - & & \\
\hline \multicolumn{8}{|c|}{ Panel C - Reconocimiento Oportuno de Pérdidas } \\
\hline & 4571 & & 0.2807 & & + & & \\
\hline \multicolumn{8}{|c|}{ Panel D - Relevancia Valorativa } \\
\hline $\begin{array}{l}\text { Modelo de Ohlson } \\
\text { (1995) }\end{array}$ & $\beta_{1}$ & $\beta_{2}$ & $\beta_{3}$ & $\beta_{4}$ & $\beta_{5}$ & $\begin{array}{l}\text { R2 } \\
\text { Adj. }\end{array}$ & Obs. \\
\hline Coeficiente & 0.471 & 0.345 & 1.377 & 0.001 & -0.019 & 0.49 & 2567 \\
\hline Signo esperado & + & + & & + & + & & \\
\hline $\begin{array}{l}\text { Nivel de signifi- } \\
\text { cancia }\end{array}$ & $\#$ & \#\# & \#\# & $\#$ & $\#$ & & \\
\hline
\end{tabular}

Modelo de Bartov et

al. (2005)

\begin{tabular}{lllll}
$\beta_{1}$ & $\beta_{2}$ & $\beta_{3}$ & R2 & Obs. \\
\hline 0.358 & -0.136 & -0.001 & Adj. & \\
\hline
\end{tabular}

Signo esperado 
Nivel de signifi- \#

cancia

\begin{tabular}{lllllllllll}
\hline $\begin{array}{l}\text { Modelo de } \\
\text { Basu (1997) }\end{array}$ & $\beta_{1}$ & $\beta_{2}$ & $\beta_{3}$ & $\beta_{4}$ & $\beta_{5}$ & $\beta_{6}$ & $\beta_{7}$ & $\begin{array}{l}\text { R2 } \\
\text { Odj. }\end{array}$ \\
\cline { 2 - 10 } & & & & & & & & & Obs. \\
Coeficiente & 0.008 & -0.097 & 0.032 & -0.008 & 0.004 & 0.037 & -0.036 & 0.13 & 2567 \\
Signo esperado & + & & + & & + & & + & & \\
Nivel de signifi- & $\#$ & $\# \#$ & $\# \# \#$ & & & & & & \\
cancia & & & & & & & & & \\
\hline
\end{tabular}

Fuente: Elaboración propia

$*, * *, * * *$ Indica diferencia estadísticamente significativa entre periodos con un nivel $p<0.01, p<0.05, p<0.1$ respectivamente (prueba de una cola). La significatividad estadística de la diferencia entre periodos para cada métrica se obtiene a través de la prueba t-test aplicado sobre la distribución empírica de las diferencias (bootstrapping).

\#, \#\#, \#\#\# Indica diferencia significativa de cero (0) con un nivel $p<0.1, p<0.05, p<0.01$ respectivamente (prueba de dos colas). La significatividad estadística distinta de cero se obtiene a través de la significatividad de cada coeficiente reportado.

Es tabulado los coeficientes SPO (Panel B) y LNEG (Panel C) obtenidos a través del modelo LOGIT.

Para todas las regresiones, excepto las basadas en modelos LOGIT, se evalúa los supuestos del modelo de regresión lineal bajo mínimos cuadrados ordinarios (MCO); no se evidencia multicolinealidad entre las regresoras de los modelos, obteniéndose los respectivos Factores de Inflación de la Varianza (VIF) con valores menores a 5 y algunos cuantos menores a 10. Se asume el supuesto de homocedasticidad regresando todas las ecuaciones bajo MCO con el test Robusto de White en STATA. Los test de Durbin-Watson y de Durbin indican alta probabilidad de falsedad en la hipótesis de no autocorrelación (probabilidad mayor a 0.10) en los modelos de la Varianza del $\Delta \mathrm{NI} *$ para las observaciones pertenecientes al periodo de GAAP Locales (Panel A), de Ohlson (1995) (Panel D) y de Bartov et al. (2005) (Panel D). Esta situación se corrige realizando nuevamente la regresión robusta bajo el modelo Prais-Winsten en el mismo software (utiliza el modelo de mínimos cuadrados generalizados MCG). Para las regresiones del Panel A, a pesar que el supuesto de normalidad en las perturbaciones no es confirmado con el test de Shapiro-Wilk, este supuesto se relaja debido a que el tamaño de la muestra es lo suficientemente grande (superior a 30 observaciones), por tanto se asume normalidad de acuerdo con el Teorema Central del Límite. En todas las variables de las regresiones, exceptuando las variables dicótomas, se controla los efectos de los valores outliers (winsorized) en los percentiles 5 y 95. Los residuales del Panel A, son obtenidos a través del modelo de mínimos cuadrados ordinarios (MCO), los valores outliers de los residuales son controlados (winsorized) en los percentiles 1 y 99.

El Panel A de la Tabla 5, reporta para el periodo de post-adopción, una significativa disminución en las expresiones relacionadas con la variabilidad del cambio en el resultado contable $\left(\Delta \mathrm{NI}^{*}\right)$ y el ratio de la variabilidad $\left(\Delta \mathrm{NI}^{*}\right) /$ la variabilidad $(\Delta \mathrm{CFO} *)(0.0017$ vs. 0.0027$)$ y (0.1998 vs. 0.2562$)$ respectivamente, sugiriendo mayor alisamiento del resultado para los periodos de aplicación de IFRS, sin embargo, la Correlación de ACC* y CFO* resulta tener un aumento estadísticamente no significativo durante este último periodo (-0.6966 vs. -0.7248).

Respecto a la frecuencia en el reporte de utilidades mínimas (Panel B) y a la frecuencia en el reporte de grandes pérdidas (Panel C), los hallazgos no sugieren un cambio estadísticamente significativo entre periodos (SPOS=-0.1362) y (LNEG=-0.1689) respectivamente; de acuerdo con esto, los resultados obtenidos en el Panel C permiten rechazar nuevamente la segunda hipótesis. 
Con referencia al Panel D, el modelo propuesto por Ohlson (1995) presenta un coeficiente negativo y significativo para la magnitud incremental del valor en libros del patrimonio $\left(\beta_{4}=-0.0087\right)$, al igual que para la magnitud incremental del resultado contable durante el periodo de aplicación de IFRS ( $\left.\beta_{5}=-0.0615\right)$, sin embargo, la disminución en la capacidad informativa del resultado contable, no es corroborado a través del modelo propuesto por Bartov et al. (2005) $\left(\beta_{3}=-0.005\right)$ dado que no reporta significancia estadística. En términos agregados, los resultados sugieren menor capacidad explicativa del valor en libros del patrimonio y del resultado contable ante las variaciones en el precio por acción, respecto al periodo de inmediatamente anterior a la aplicación de IFRS.

Finalmente, el coeficiente de malas noticias bajo el modelo propuesto por Basu (1997), a pesar de ser positivo no reporta significancia estadística durante el periodo de post-adopción ( $\beta_{7}=0.057$ ), tampoco reporta significancia para el periodo previo a la aplicación de las IFRS $=\left(\beta_{3}-0.008\right)$; estos resultados sugieren que durante el periodo de aplicación de IFRS (LOCAL GAAP), las rentabilidades negativas se incorporan con igual oportunidad en el resultado contable respecto a las buenas noticias, no evidenciándose cambios en la relevancia valorativa durante el periodo de aplicación de IFRS.

Los hallazgos obtenidos para Latinoamérica y el Caribe, sugieren una disminución en el nivel de la calidad contable durante el periodo inmediatamente siguiente a la aplicación de las IFRS, visto desde un aumento en la manipulación del resultado y desde una disminución en la capacidad informativa de las magnitudes contables en los mercados de capitales (relevancia valorativa); las conclusiones obtenidas para estos países durante el periodo inmediatamente siguiente a la aplicación de las IFRS, aparte de rechazar la primera y tercera hipótesis, distan de las conclusiones obtenidas para esta misma región bajo un análisis de 5 años de aplicación de IFRS aproximadamente (Tabla 4).

Analizando conjuntamente los resultados obtenidos (Tablas 4 y 5), los hallazgos permiten concluir que la familiarización con el uso de las IFRS durante varios años de aplicación, impulsan al mejoramiento de la calidad contable para los países latinos y del Caribe, constatado a través de un mejoramiento en los atributos contables (disminución en la manipulación del resultado); sin embargo, y a pesar del uso de las IFRS por varios años, no se consigue que la información contable de calidad adquiera mayor relevancia valorativa (atributos de mercado) para los actores en estos mercados de capitales. Estos resultados también están parcialmente conforme a lo documentado por Iatridis et al. (2010) para el caso griego, solamente en lo referente a que la familiarización con las IFRS durante varios años disminuye el uso de la manipulación del resultado ejercida por los directivos. 
Tabla 5

Comparación de la calidad contable sólo periodo previo y periodo posterior a las IFRS (muestra restringida)

\begin{tabular}{|c|c|c|c|c|c|c|c|c|c|}
\hline & \multicolumn{2}{|c|}{ Obs. } & \multicolumn{2}{|c|}{ Pre-adopción } & \multicolumn{2}{|c|}{$\begin{array}{l}\text { Post-adopción } \\
\text { (IFRS) }\end{array}$} & $\begin{array}{l}\text { Signo } \\
\text { Esperado }\end{array}$ & \multicolumn{2}{|c|}{$\begin{array}{r}\text { Diferencia } \\
\text { (Post-Pre) }\end{array}$} \\
\hline & Pre & Post & & & & & & & \\
\hline \multicolumn{10}{|c|}{ Panel A-Alisamiento del Resultado } \\
\hline $\begin{array}{l}\text { Varianza } \\
\text { del } \Delta \mathrm{NI}^{*}\end{array}$ & 699 & 699 & 0.002 & & 0.0017 & & + & & $-0.0001^{*}$ \\
\hline $\begin{array}{l}\text { Varianza } \\
\text { del } \Delta \mathrm{NI}^{*} / \Delta \mathrm{CFO}^{*}\end{array}$ & 699 & 699 & 0.256 & & 0.1998 & & + & & $-0.0564 * *$ \\
\hline $\begin{array}{l}\text { Correlación de } \mathrm{ACC}^{*} \text { y } \\
\mathrm{CFO} *\end{array}$ & 699 & 699 & -0.72 & & -0.696 & & + & & 0.0282 \\
\hline \multicolumn{10}{|c|}{ Panel B - Manipulación hacia un Resultado Objetivo } \\
\hline$\beta_{11} S P O S$ & 139 & & & -0.1362 & & & - & & \\
\hline \multicolumn{10}{|c|}{ Panel C - Reconocimiento Oportuno de Pérdidas } \\
\hline$\beta_{11} L N E G$ & 139 & & & -0.1689 & & & + & & \\
\hline \multicolumn{10}{|c|}{ Panel D - Relevancia Valorativa } \\
\hline $\begin{array}{l}\text { Modelo de Ohlson } \\
\text { (1995) }\end{array}$ & $\beta_{1}$ & & $\beta_{2}$ & $\beta_{3}$ & $\beta_{4}$ & & $\beta_{5}$ & $\mathrm{R} 2 \Lambda \mathrm{dj}$ & Obs. \\
\hline Coeficiente & 0.5746 & & .1945 & 2.0804 & -0.008 & & -0.0615 & 0.56 & 694 \\
\hline Signo esperado & + & & + & & + & & + & & \\
\hline Nivel de significancia & $\#$ & & & & \#\# & & $\#$ & & \\
\hline $\begin{array}{l}\text { Modelo de Bartov et al } \\
\text { (2005) }\end{array}$ & $\beta_{1}$ & & $\beta_{2}$ & & $\beta_{3}$ & & & R2 Adj. & Obs. \\
\hline Coeficiente & 0.372 & & 0.235 & & -0.0 & & & 0.22 & 694 \\
\hline Signo esperado & + & & + & & + & & & & \\
\hline Nivel de significancia & $\#$ & & & & & & & & \\
\hline $\begin{array}{l}\text { Modelo de Basu } \\
\text { (1997) }\end{array}$ & $\beta_{1}$ & $\beta_{2}$ & $\beta_{3}$ & $\beta_{4}$ & $\beta_{5}$ & $\beta_{6}$ & $\beta_{7}$ & $\begin{array}{l}\text { R2 } \\
\text { Adj. }\end{array}$ & Obs. \\
\hline Coeficiente & 0.013 & -0.022 & -0.008 & 0.055 & -0.001 & 0.058 & 0.057 & 0.09 & 694 \\
\hline Signo esperado & + & & + & & + & & + & & \\
\hline Nivel de significancia & $\#$ & & & & & & & & \\
\hline
\end{tabular}

Fuente: Elaboración propia

$*, * *, * * *$ Indica diferencia estadísticamente significativa entre periodos con un nivel $p<0.01, p<0.05, p<0.1$ respectivamente (prueba de una cola). La significatividad estadística de la diferencia entre periodos para cada métrica se obtiene a través de la prueba t-test aplicado sobre la distribución empírica de las diferencias (bootstrapping).

\#, \#\#, \#\#\# Indica diferencia significativa de cero (0) con un nivel $p<0.1, p<0.05, p<0.01$ respectivamente (prueba de dos colas). La significatividad estadística distinta de cero se obtiene a través de la significatividad de cada coeficiente reportado.

Es tabulado los coeficientes SPO (Panel B) y LNEG (Panel C) obtenidos a través del modelo LOGIT.

Para todas las regresiones, excepto las basadas en modelos LOGIT, se evalúa los supuestos del modelo de regresión lineal bajo mínimos cuadrados ordinarios (MCO); no se evidencia multicolinealidad entre las regresoras de los modelos, obteniéndose los respectivos Factores de Inflación de la Varianza (VIF) con valores menores a 5 y algunos cuantos menores a 10. Se asume el supuesto de homocedasticidad regresando todas las ecuaciones bajo MCO con el test Robusto de White en STATA. Los test de Durbin-Watson 
y de Durbin indican alta probabilidad de falsedad en la hipótesis de no autocorrelación (probabilidad mayor a 0.10) en los modelos de $\triangle \mathrm{CFO} *$ necesaria para calcular el indicador de la Varianza $\Delta \mathrm{NI} * / \Delta \mathrm{CFO} *$ para las observaciones pertenecientes al periodo de IFRS (Panel A) y de Ohlson (1995) (Panel D). Esta situación se corrige realizando nuevamente la regresión robusta bajo el modelo Prais-Winsten en el mismo software (utiliza el modelo de mínimos cuadrados generalizados MCG). Para las regresiones del Panel A, a pesar que el supuesto de normalidad en las perturbaciones no es confirmado con el test de Shapiro-Wilk, este supuesto se relaja debido a que el tamaño de la muestra es lo suficientemente grande (superior a 30 observaciones), por tanto se asume normalidad de acuerdo con el Teorema Central del Límite. En todas las variables de las regresiones, exceptuando las variables dicótomas, se controla los efectos de los valores outliers (winsorized) en los percentiles 5 y 95. Los residuales del Panel A, son obtenidos a través del modelo de mínimos cuadrados ordinarios (MCO); los valores outliers de los residuales son controlados (winsorized) en los percentiles 1 y 99 .

\section{Análisis de sensibilidad}

Teniendo en cuenta que más del $60 \%$ y $70 \%$ de las observaciones en las Muestras A y B respectivamente, pertenecen a empresas domiciliadas y cotizadas en Brasil y Chile, son aplicados nuevamente los indicadores de medición de la calidad contable para cada una de estas naciones, asimismo son considerados estos indicadores para el resto de países analizados conjuntamente ${ }^{1}$. Para el caso brasileño, los resultados obtenidos en términos agregados, no difieren de los reportados en la Tabla 4, sin embargo, cabe resaltar que la capacidad informativa del resultado contable y del valor en libros del patrimonio en el mercado de capitales brasileño (Modelos de Ohlson y Bartov), disminuye significativamente durante el periodo de aplicación de las IFRS.

Al considerar el caso chileno, si bien los resultados no difieren de los conseguidos en los paneles B al D de la Tabla 4, si indican un incremento significativo en el nivel de manipulación del resultado dado que dos de los tres indicadores relacionados con el nivel de alisamiento resultan ser negativos y significativos en términos estadísticos. Finalmente, abordando el resto de países latinoamericanos y del Caribe, los resultados obtenidos permiten no diferir de las conclusiones alcanzadas a partir de la Tabla 4 , asimismo los resultados contribuyen adicionalmente, al mejoramiento en los atributos contables para dichos países dado un incremento significativo en la frecuencia al reconocer grandes pérdidas (LNEG) durante el periodo de aplicación de IFRS.

\section{Conclusiones}

Esta investigación tiene por objetivo examinar si la calidad del reporte financiero (expresión de medición de la calidad contable) ha incrementado después de la aplicación de las IFRS para las compañías domiciliadas y cotizadas en los mercados de capitales latinoamericanos y del Caribe. Específicamente, se examina si la aplicación de las IFRS en 16 países latinoamericanos y 4 países del Caribe es asociada con menor manipulación del resultado, mayor oportunidad

\footnotetext{
${ }^{1}$ Únicamente se describen los resultados obtenidos de estos análisis individuales sin reportarse tablas y anexos relacionados con la aplicación de los indicadores de medición propuestos en la metodología.
} 
en el reconocimiento de pérdidas y mayor relevancia valorativa de la información contable durante un periodo de análisis de 11 años aproximadamente (2006 a 2016).

Después de controlar los factores específicos de las compañías, del tiempo y del ambiente económico que afectan la magnitud de las cifras contables, los resultados sugieren que durante el primer periodo de aplicación de IFRS, se presenta una disminución en la calidad contable para América Latina y el Caribe, vista desde un incremento en manipulación del resultado y una disminución en la relevancia valorativa de las cifras contables para los inversores en los mercados de capitales; sin embargo, al replicar el análisis incluyendo varios años de aplicación de IFRS, únicamente se percibe un mejoramiento en los atributos contables (disminución en el nivel de manipulación del resultado), mientras que la utilidad de la información contable en los mercados latinos y del Caribe permanecen sin ser valorados con mayor utilidad.

El uso de la metodología propuesta por Barth et al. (2008) y empleada en esta investigación, si bien no es un indicador perfecto del concepto multifacético de la calidad contable, permite comparar y contrastar los resultados obtenidos para Latinoamérica y el Caribe con los obtenidos para otros países bajo esta misma metodología. Dichos resultados, tal y como lo documentan investigaciones previas, no sólo dependen de los estándares contables IFRS, es un efecto combinado además de los estándares, de los demás elementos del sistema de información contable, como lo son: los mecanismos de refuerzo (Enforcement) para garantizar la calidad de la información, guías de aplicación e interpretación de las IFRS y el riesgo de litigio que recae en varias economías sobre los responsables de la preparación y aseguramiento de la información financiera (Burgstahler et al., 2006; Cairns, 1999).

Las inferencias y conclusiones obtenidas de esta investigación parten de la presunción realizada por Barth et al. (2008), un mayor alisamiento del resultado se traduce en mayor manipulación del mismo (baja calidad contable); siendo importante aclarar que el uso e interpretación de los indicadores de medición de la calidad contable, dependen de los atributos de la misma que se desea evaluar, por ejemplo, otro tipo de investigaciones (Francis et al. 2008; Ewert y Wagenhofer 2011), ha utilizado el incremento en el alisamiento del resultado (earnings smoothing) como indicativo de mayor previsibilidad para los inversores (mayor calidad contable) a través de la incorporación de información adicional en el resultado contable por parte de los gerentes, consiguiendo un mayor alisamiento del mismo.

Este articulo contribuye a la literatura incluyendo a casi la totalidad de los países latinoamericanos y del Caribe durante un amplio periodo de análisis, aportando suficiente evidencia sobre las implicaciones del uso de las IFRS en la calidad del reporte financiero para países con sistema contable de influencia francesa (La Porta et al., 1997), resolviendo las limitaciones identificadas en la literatura previa, en que los resultados no podrían generalizarse dado que sólo se disponía información para un solo país y en un rango limitado de años de aplicación de IFRS. 
Futuras investigaciones podrían comparar el impacto en la calidad contable entre los mismos países latinoamericanos, con medidas adicionales de la calidad basadas por ejemplo, en la discrecionalidad ejercida por la gerencia sobre las cifras no efectivas (ajustes por devengo) para manipular el resultado, incluso comparar la calidad contable entre los países latinoamericanos (sistema contable de origen francés) respecto a países con sistemas contables de origen anglosajón, escandinavo y/o germánico (La Porta et al., 1997).

\section{Referencias Bibliográficas}

Abdullah, W. R. W., Maruhun, E. N. S., \& Tarmizi, M. A. (2017). The Adoption of IFRS and Earnings Management: Evidence from The Construction Industry in Malaysia. Global Conference on Business and Economics Research, August(n.d.), 1-8. Disponible en: https://econ.upm.edu.my/upload/dokumen/20171011154330051-_ WAN_RAZALILA.pdf y Consultado: 15/08/2019.

Ahmed, A. S., Neel, M., \& Wang, D. (2013). Does mandatory adoption of IFRS improve accounting quality? Preliminary evidence. Contemporary Accounting Research, 30(4), 1344-1372. http://doi.org/10.1111/j.19113846.2012.01193.x

Armstrong, C. S., Barth, M. E., Jagolinzer, A. D., \& Riedl, E. J. (2010). Market reaction to the adoption of IFRS in Europe. The accounting review, 85(1), 31-61. http://doi.org/10.2308/accr.2010.85.1.31

Aubert, F., \& Grudnitski, G. (2011). The impact and importance of mandatory adoption of international financial reporting standards in Europe. Journal of International Financial Management \& Accounting, 22(1), 1-26. http://doi.org/10.1111/j.1467-646X.2010.01043.x

Ball, R. (2001). Infrastructure requirements for an economically efficient system of public financial reporting and disclosure. Brookings-Wharton Papers on Financial Services, 2001(1), 127-169. http://doi.org/10.1353/ pfs.2001.0002

Ball, R., Robin, A., \& Wu, J. S. (2003). Incentives versus standards: properties of accounting income in four East Asian countries. Journal of accounting and economics, 36(1-3), 235-270. http://doi.org/10.1016/j.jacceco.2003.10.003

Ball, R., \& Shivakumar, L. (2005). Earnings quality in UK private firms: comparative loss recognition timeliness. Journal of accounting and economics, 39(1), 83-128. http://doi.org/10.1016/j.jacceco.2004.04.001

Barth, M. E., Landsman, W. R., \& Lang, M. H. (2008). International accounting standards and accounting quality. Journal of Accounting Research, 46(3), 467-498. http://doi.org/10.1111/j.1475-679X.2008.00287.x

Barth, M. E., Landsman, W., Lang, M., \& Williams, C. (2006). Accounting quality: International accounting standards and US GAAP. Manuscript, Stanford University. 1-46. Disponible en: http://public.kenan-flagler.unc. edu/faculty/langm/bllw_0302.pdf y Consultado: 15/08/2019.

Bartov, E., Goldberg, S. R., \& Kim, M. (2005). Comparative value relevance among german, US, and international accounting standards: A german stock market perspective. Journal of Accounting, Auditing \& Finance, 20(2), 95-119. https://doi.org/10.1177/0148558X0502000201

Basu, S. (1997). The conservatism principle and the asymmetric timeliness of earnings. Journal of Accounting and Economics, 24(1), 3-37. http://doi.org/10.1016/S0165-4101(97)00014-1

Brad, L., Dobre, F., Ţurlea, C., \& Braşoveanu, I. V. (2014). The impact of IFRS adoption in Romania upon the earnings management of the Bucharest stock exchange entities. Procedia Economics and Finance, 15, 871-876. http://doi.org/10.1016/S2212-5671(14)00550-4

Burgstahler, D. C., Hail, L., \& Leuz, C. (2006). The importance of reporting incentives: Earnings management in European private and public firms. The Accounting Review, 81(5), 983-1016. http://doi.org/10.2308/ accr.2006.81.5.983 
Burgstahler, D., \& Dichev, I. (1997). Earnings management to avoid earnings decreases and losses. Journal of Accounting and Economics, 24(1), 99-126. http://doi.org/10.1016/S0165-4101(97)00017-7

Bushman, R. M., Piotroski, J. D., \& Smith, A. J. (2004). What determines corporate transparency?. Journal of accounting research, 42(2), 207-252. http://doi.org/10.1111/j.1475-679X.2004.00136.x

Bushman, R. M., \& Piotroski, J. D. (2006). Financial reporting incentives for conservative accounting: The influence of legal and political institutions. Journal of Accounting and Economics, 42(1-2), 107-148. http://doi. org/10.1016/j.jacceco.2005.10.005

Cahan, S. F., Emanuel, D., \& Sun, J. (2009). The effect of earnings quality and country-level institutions on the value relevance of earnings. Review of Quantitative Finance and Accounting, 33(4), 371. http://doi.org/10.1007/ s11156-009-0117-z

Cairns, D. (1999). Degrees of compliance. Accountancy International, 124(1273), 114-118.

Capkun, V., Collins, D., \& Jeanjean, T. (2016). The effect of IAS/IFRS adoption on earnings management (smoothing): A closer look at competing explanations. Journal of Accounting and Public Policy, 35(4), 352-394. http:// doi.org/10.1016/j.jaccpubpol.2016.04.002

Cardona Montoya, J. (2018). Impacto de las IFRS en la calidad del reporte financiero en Latinoamérica y el Caribe. Contaduría y Administración, 63(SPE2), 841-861. http://doi.org/10.22201/fca.24488410e.2018.1233

Cardona Montoya, J. C., \& Cano Morales, A. M. (2017). The impact of international financial reporting standards on canadian accounting quality. Espacios, 38(52). Disponible en: https://www.revistaespacios.com/ a17v38n52/17385218.html y consultado: 15/08/2019.

Christensen, H. B., Lee, E., Walker, M., \& Zeng, C. (2015). Incentives or standards: What determines accounting quality changes around IFRS adoption?. European Accounting Review, 24(1), 31-61. http://doi.org/10.1080/0 9638180.2015.1009144

Chua, Y. L., Cheong, C. S., \& Gould, G. (2012). The impact of mandatory IFRS adoption on accounting quality: Evidence from Australia. Journal of International Accounting Research, 11(1), 119-146. http://doi.org/10.2308/ jiar-10212

Conesa, I. M., Manzano, M. P., \& Sánchez, H. G. (2011). La calidad del resultado pre y post-adaptación a las IFRS en México por el CINIF. Disponible en http://www.aeca1.org/pub/on_line/comunicaciones_aal2011/cd/54a.pdf y consultado: 15/08/2019.

da Silva, J. P., Borges, T. J. G., de Souza Gonçalves, R., \& do Nascimento, D. V. R. (2017). Convergence to IFRS standard and smoothing results in electric power companies. BASE-Revista de Administração e Contabilidade da Unisinos, 14(4), 281-296. http://doi.org/10.4013/base.2017.144.04

Devalle, A., Onali, E., \& Magarini, R. (2010). Assessing the value relevance of accounting data after the introduction of IFRS in Europe. Journal of International Financial Management \& Accounting, 21(2), 85-119. http:// doi.org/10.1111/j.1467-646X.2010.01037.x

Dimitropoulos, P. E., Asteriou, D., Kousenidis, D., \& Leventis, S. (2013). The impact of IFRS on accounting quality: Evidence from Greece. Advances in Accounting, 29(1), 108-123. http://doi.org/10.1016/j.adiac.2013.03.004

Ebrahim, A. (2007). Earnings management and board activity: an additional evidence. Review of Accounting and Finance, 6(1), 42-58. http://doi.org/10.1108/14757700710725458

Ewert, R., \& Wagenhofer, A. (2011). Earnings quality metrics and what they measure. SSRN Electronic Journal, 1-62. http://doi.org/10.2139/ssrn.1697042

Francis, J. R., Khurana, I. K., \& Pereira, R. (2003). The role of accounting and auditing in corporate governance and the development of financial markets around the world. Asia-Pacific Journal of Accounting \& Economics, 10(1), 1-30. http://doi.org/10.1080/16081625.2003.10510613

Francis, J., Olsson, P., \& Schipper, K. (2008). Earnings quality. Foundations and Trends $®$ in Accounting, 1(4), 259340. http://doi.org/10.1561/1400000004 
Fuad, \& Wijanarto, W. T. (2017). How mandatory IFRS adoption changes firms' opportunistic behavior: empirical evidences from the earnings management perspective. Academy of Accounting and Financial Studies Journal, 21(2), 1-10. Disponible en: https://www.abacademies.org/journals/month-august-year-2017-vol-21-issue2-journal-aafsj-past-issue.html y Consultado: 15/08/2019.

Günther, N., Gegenfurtner, B., Kaserer, C., \& Achleitner, A. (2009). International financial reporting standards and earnings quality: The myth of voluntary vs. mandatory adoption. CEFS Working Paper No. 2009-09. 1-44. http://doi.org/10.2139/ssrn.1413145

Hail, L., Leuz, C., \& Wysocki, P. (2010). Global accounting convergence and the potential adoption of IFRS by the US (Part I): Conceptual underpinnings and economic analysis. Accounting Horizons, 24(3), 355-394. http:// doi.org/10.2308/acch.2010.24.3.355

Halabi, H., \& Yi, L. (2015). Mandatory IFRS adoption and earnings quality: The impact of country-specific factors. European Financial Management Association 2015 Annual Meetings. Disponible en: https://repository.hkbu. edu.hk/hkbu_staff_publication/4844/ y Consultado: 16/08/2019.

Healy, P. M., \& Wahlen, J. M. (1999). A review of the earnings management literature and its implications for standard setting. Accounting Horizons, 13(4), 365-383. https://doi.org/10.2308/acch.1999.13.4.365.

Hellström, K. (2006). The value relevance of financial accounting information in a transition economy: The case of the Czech Republic. European Accounting Review, 15(3), 325-349. http://doi.org/10.1080/09638180600916242

Houqe, M. N., van Zijl, T., Dunstan, K., \& Karim, A. W. (2012). The effect of IFRS adoption and investor protection on earnings quality around the world. The International journal of accounting, 47(3), 333-355. http://doi. org/10.1016/j.intacc.2012.07.003

Hove, M. R. (1990). The Anglo-American influence on international accounting standards: The case of the disclosure standards of the international accounting standards committee. In R. S. O. Wallace, J. M. Samuels, \& R. J. Briston (Eds.), Research in third world accounting (Vol. 1, pp. 55-66). London, England: JAI Press.

Iatridis, G. (2008). Implementation of international financial reporting standards and the quality of financial statement information; an investigation of earnings management and value relevance. Working paper, University of Thessaly. http://doi.org/10.1.1.496.9261

Iatridis, G. (2010). International financial reporting standards and the quality of financial statement information. International Review of Financial Analysis, 19(3), 193-204. http://doi.org/10.1016/j.irfa.2010.02.004

Iatridis, G., \& Rouvolis, S. (2010). The post-adoption effects of the implementation of international financial reporting standards in Greece. Journal of International Accounting, Auditing and Taxation, 19(1), 55-65. http://doi. org/10.1016/j.intaccaudtax.2009.12.004

Isidro, H., Nanda, D., \& Wysocki, P. D. (2016). Financial Reporting Differences Around the World: What Matters? SSRN Electronic Journal. http://doi.org/10.2139/ssrn.2788741

Klann, R. C., \& Beuren, I. M. (2015). The impact of the international accounting convergence on income smoothing in brazillian companies. Brazilian Business Review, 12(2), 1-24. http://doi.org/10.15728/bbr.2015.12.2.1

Klein, A. (2002). Audit committee, board of director characteristics, and earnings management. Journal of accounting and economics, 33(3), 375-400. http://doi.org/10.1016/S0165-4101(02)00059-9

Kothari, S. P. (2000). The role of financial reporting in reducing financial risks in the market. Conference Series-Federal Reserve Bank of Boston, 44(n.d.), 89-112. Disponible en: https://econpapers.repec.org/article/fipfedbcp/y_3a2000_3ai_3ajun_3ap_3a89-112_3an_3a44.htm y Consultado: 16/08/2019.

Land, J., \& Lang, M. H. (2002). Empirical evidence on the evolution of international earnings. The Accounting Review, 77(s-1), 115-133. http://doi.org/10.2308/accr.2002.77.s-1.115

Lang, M., Raedy, J. S., \& Wilson, W. (2006). Earnings management and cross listing: Are reconciled earnings comparable to US earnings?. Journal of Accounting and Economics, 42(1-2), 255-283. http://doi.org/10.1016/j. jacceco.2006.04.005

Lang, M., Raedy, J. S., \& Yetman, M. H. (2003). How representative are firms that are cross-listed in the United States? An analysis of accounting quality. Journal of Accounting Research, 41(2), 363-386. http://doi. org/10.1111/1475-679X.00108 
La Porta, R., Lopez DeSilanes, F., Shleifer, A., \& Vishny, R. W. (1997). Legal determinants of external finance. Journal of Finance, 52(3), 1131-1150. http://doi.org/10.1111/j.1540-6261.1997.tb02727.x

La Porta, R., Lopez-de-Silanes, F., Shleifer, A., \& Vishny, R. (2000). Investor protection and corporate governance. Journal of financial economics, 58(1-2), 3-27. http://doi.org/10.1016/S0304-405X(00)00065-9

La Porta, R., Lopez-de-Silanes, F., Pop-Eleches, C., \& Shleifer, A. (2004). Judicial checks and balances. Journal of Political Economy, 112(2), 445-470. http://doi.org/10.1086/381480

Leuz, C. (2010). Different approaches to corporate reporting regulation: How jurisdictions differ and why. Accounting and business research, 40(3), 229-256. http://doi.org/10.1080/00014788.2010.9663398

Leuz, C., Nanda, D., \& Wysocki, P. D. (2003). Earnings management and investor protection: An international comparison. Journal of Financial Economics, 69(3), 505-527. http://doi.org/10.1016/S0304-405X(03)00121-1

Leuz, C., \& Wysocki, P. D. (2016). The economics of disclosure and financial reporting regulation: Evidence and suggestions for future research. Journal of Accounting Research, 54(2), 525-622. http://doi.org/10.1111/1475-679X.12115

Lin, S., Riccardi, W., \& Wang, C. (2012). Does accounting quality change following a switch from US GAAP to IFRS? Evidence from Germany. Journal of Accounting and Public Policy, 31(6), 641-657. http://doi. org/10.1016/j.jaccpubpol.2012.10.006

Liu, C., Yao, L. J., Hu, N., \& Liu, L. (2011). The impact of IFRS on accounting quality in a regulated market: An empirical study of China. Journal of Accounting, Auditing \& Finance, 26(4), 659-676. http://doi. org/10.1177/0148558X11409164

Melgarejo, M. (2017). Additional Evidence on the Impact of the International Financial Reporting Standards on Earnings Quality: Evidence from Latin America. The Journal of Applied Business and Economics, 19(7), 4959. https://doi.org/10.33423/jabe.v19i7.740

Ohlson, J. A. (1995). Earnings, book values, and dividends in equity valuation. Contemporary Accounting Research, 11(2), 661-687. http://doi.org/10.1111/j.1911-3846.1995.tb00461.x

Paananen, M. (2008). The IFRS adoption's effect on accounting quality in Sweden. SSRN Electronic Journal. http://doi.org/10.2139/ssrn.1097659

Paananen, M., \& Lin, H. (2009). The development of accounting quality of IAS and IFRS over time: The case of Germany. Journal of International Accounting Research, 8(1), 31-55. http://doi.org/10.2308/jiar.2009.8.1.31

Palacios Manzano, M., \& Martinez Conesa, I. (2014). Assessing the impact of IFRS adaptation on earnings management: An emerging market perspective. Transformation in Business \& Economics, 13(1), 21-40. Disponible en: http://www.transformations.knf.vu.lt/31/ge31.pdf y consultado: 16/08/2019.

Peasnell, K. V., Pope, P. F., \& Young, S. (2005). Board monitoring and earnings management: Do outside directors influence abnormal accruals?. Journal of Business Finance \& Accounting, 32(7-8), 1311-1346. http://doi.org/10.1111/j.0306-686X.2005.00630.x

Pelucio-Grecco, M. C., Geron, C. M. S., Grecco, G. B., \& Lima, J. P. C. (2014). The effect of IFRS on earnings management in Brazilian non-financial public companies. Emerging Markets Review, 21, 42-66. http://doi. org/10.1016/j.ememar.2014.07.001

Piot, C., Janin, R., \& Dumontier, P. (2010). IFRS consequences on accounting conservatism within Europe. CAAA Annual Conference 2011. http://doi.org/10.2139/ssrn.1724635

Porta, R. L., Lopez-de-Silanes, F., Shleifer, A., \& Vishny, R. W. (1998). Law and finance. Journal of political economy, 106(6), 1113-1155. http://doi.org/10.1086/250042

Reverte, C. (2008). Institutional differences in EU countries and their relationship with earnings management differences: Implications for the pan-European stock market. Journal of Accounting \& Organizational Change, 4(2), 182-200. http://doi.org/10.1108/18325910810878964

Santana, V. d. F., Rathke, A.A.T., Laurenço, I., \& Dalmmcio, F.Z. (2014). IFRS accounting quality in Latin America: A comparison with Anglo-Saxon and continental European countries and the role of cross listing in the US. SSRN Electronic Journal, 1-24. Disponible en: http://ssrn.com/abstract=2438268 y Consultado: 15/08/2019. 
Schipper, K. (1989). Commentary on earnings management. Accounting Horizons, 3(4), 91-102. Disponible en: https://search.proquest.com/openview/177246e104b43553542ab048997f1a4e/1?pq-origsite=gscholar\&cbl=3330 y Consultado: 16/08/2019.

Temiz, H., \& Güleç, Ö. F. (2017). Mandatory adoption of IFRS in emerging markets: the case of Turkey. Accounting and Management Information Systems, 16(4), 560-580. http://doi.org/10.24818/jamis.2017.04007

Trueman, B., \& Titman, S. (1988). An explanation for accounting income smoothing. Journal of Accounting Research, 26(Supl.), 127-139. http://doi.org/10.2307/2491184

Vieira, R., Martins, V., Machado, A., \& Domingues, J. (2011). Impacts of partial adoption of IFRS in Brazil: Effects on financial information quality of publicly traded companies. British Journal of Economics, Finance and Management Sciences, 1(2), 96-112. Disponible en: http://www.ajournal.co.uk/EFArticles1(2).htm y Consultado: $15 / 08 / 2019$.

World Economic Forum. (2017). The Global competitiveness report 2017-2018. Disponible en: http://reports.weforum.org/global-competitiveness-index-2017-2018 y Consultado: 15/08/2019.

Wysocki, P. (2011). New institutional accounting and IFRS. Accounting and business research, 41(3), 309-328. http://doi.org/10.1080/00014788.2011.575298

Zeghal, D., Chtourou, S. M., \& Fourati, Y. M. (2012). The effect of mandatory adoption of IFRS on earnings quality: Evidence from the European Union. Journal of International Accounting Research, 11(2), 1-25. http://doi. org/10.2308/jiar-10221

Zhou, H., Xiong, Y., \& Ganguli, G. (2009). Does the adoption of international financial reporting standards restrain earnings management? Evidence from an emerging market. Academy of Accounting and Financial Studies Journal, 13(Special Issue), 43-56. Disponible en: https://www.abacademies.org/journals/month-march-year2009-vol-13-issue-1-journal-aafsj-past-issue.html y Consultado: 15/08/2019. 